\title{
The KeY tool
}

\section{Integrating object oriented design and formal verification}

\author{
Wolfgang Ahrendt ${ }^{1}$, Thomas Baar ${ }^{2}$, Bernhard Beckert ${ }^{3}$, Richard Bubel ${ }^{4}$, Martin Giese ${ }^{1}$, Reiner Hähnle ${ }^{1}$, \\ Wolfram Menzel $^{4}$, Wojciech Mostowski ${ }^{1}$, Andreas Roth ${ }^{4}$, Steffen Schlager ${ }^{4}$, Peter H. Schmitt ${ }^{4}$ \\ ${ }^{1}$ Department of Computing Science, Chalmers University of Technology, 41296 Gothenburg, Sweden; \\ e-mail: \{ahrendt,giese,reiner,woj\}@cs.chalmers.se \\ ${ }^{2}$ Swiss Federal Institute of Technology in Lausanne, Software Engineering Laboratory, 1015 Lausanne EPFL, Switzerland; \\ e-mail: Thomas.Baar@epfl.ch \\ ${ }^{3}$ Institute for Computer Science, University of Koblenz-Landau, 56072 Koblenz, Germany; \\ e-mail: beckert@uni-koblenz.de \\ ${ }^{4}$ Department of Computer Science, University of Karlsruhe, 76128 Karlsruhe, Germany; \\ e-mail: \{bubel,menzel,aroth,schlager,pschmitt\}@ira.uka.de
}

Received: 14 December 2002/Accepted: 9 January 2004

Published online: 1 April 2004 - (C) Springer-Verlag 2004

\begin{abstract}
KeY}$ is a tool that provides facilities for formal specification and verification of programs within a commercial platform for UML based software development. Using the $\mathrm{KeY}$ tool, formal methods and object-oriented development techniques are applied in an integrated manner. Formal specification is performed using the Object Constraint Language (OCL), which is part of the UML standard. KeY provides support for the authoring and formal analysis of OCL constraints. The target language of KeY based development is JAVA CARD DL, a proper subset of JAVA for smart card applications and embedded systems. KeY uses a dynamic logic for JAVA CARD DL to express proof obligations, and provides a state-of-the-art theorem prover for interactive and automated verification. Apart from its integration into UML based software development, a characteristic feature of $\mathrm{KeY}$ is that formal specification and verification can be introduced incrementally.
\end{abstract}

Keywords: Object-oriented design - Formal specification - Formal verification - UML - OCL - Design patterns - JAVA

\section{Introduction}

$\mathrm{KeY}$ is a tool for the development of high quality objectoriented software. The "KeY" idea behind this tool is to provide facilities for formal specification and verification of programs within a software development

The research reported here has been partially supported by STINT, Vetenskapsrådet, Vinnova, and DFG. platform supporting contemporary design and implementation methodologies. The $\mathrm{KeY}$ tool empowers its users to perform formal specification and verification as part of software development based on the Unified Modeling Language (UML). To achieve this, the system is realised as the extension of a commercial UMLbased Computer Aided Software Engineering Tool (CASE tool). As a consequence, specification and verification can be performed within the extended CASE tool itself. Such a deep integration of formal specification and verification into modern software engineering concepts serves two purposes. First, formal methods and objectoriented development techniques become applicable in a meaningful combination. Second, formal specification and verification become more accessible to developers who are already using object-oriented design methodology. Moreover, $\mathrm{KeY}$ allows a lightweight usage of the provided formal techniques, as both, specification and verification, can be performed at any time, and to any desired degree. The homepage of the KeY project is http://www.key-project.org/.

The target language of KeY-driven software development is JAVA. More specifically, the verification facilities of $\mathrm{KeY}$ are restricted to code written in JAVA CARD [27, 68]. JAVA CARD is a proper subset of the JAVA programming language, excluding certain features (like threads, cloning or dynamic class loading) and with a much reduced API. The JAVA CARD language [68] and platform [69] are provided by Sun Microsystems to enable JAVA technology to run on smart cards and other devices with limited memory, such as embedded systems.

UML based software development puts an emphasis on the activity of designing the targeted system. It is in- 
creasingly accepted that the design stage is very much where one actually has the power to prevent a system from failing. This suggests that formal specification and verification should (in different ways) be closely tied to the design phase, to design documents, and to design tools. One way of combining object-oriented design and formal specification is to attach constraints to class diagrams. An appropriate notation for such a purpose is already offered by the UML: the standard [58] includes the Object Constraint Language (OCL). We briefly point out the three major roles of OCL constraints within KeY:

- The KeY tool supports the creation of constraints. While a user is free in general to formulate any desired constraint, he or she can also take advantage of the automatic generation of constraints, a feature which is realised in the $\mathrm{KeY}$ tool by extending the CASE tool's design pattern instantiation mechanism.

- The KeY tool supports the formal analysis of constraints. The relations between classes in the design imply relations between corresponding constraints, which can be analysed regardless of any implementation.

- The KeY tool supports the verification of implementations with respect to the constraints. A theorem prover with interactive and automatic operation modes can check consistency of JAVA implementations with the given constraints.

These mechanisms, and their interaction with the features already provided by the underlying CASE tool, will be described in detail in this paper.

The KeY tool realises full integration of certain formal techniques into more widely spread techniques. Nevertheless, the usage of specification or verification facilities requires additional effort and skill, which has to be motivated. In the software industry, the "residual defect ratio" (the number of bugs that remain in the shipped product) normally lies between 0.5 and 5 defects per thousand lines of non-commented source code [44]. Whether this number justifies to undertake an extra effort or not depends on the damage caused by system failures. Application areas, where this damage is known to be particularly high, include: safety critical applications (e.g. railway switches), security critical applications (e.g. access control, electronic banking), cost critical applications (which, for example, run on a large number of non-administrated devices, such as phone cards), and legally critical applications (e.g. falling under digital signature laws).

Such applications are often intended to run on smart cards or similar devices. Therefore, JAVA CARD as the target language of the $\mathrm{KeY}$ tool, is highly significant. At the same time, the technical restrictions of JAVA CARD make verification of the full language feasible. We stress that $\mathrm{KeY}$ is not restricted to being used for the development of smart card applications, because many JAVA applications do not use features excluded by JAVA CARD. In general, the $\mathrm{KeY}$ tool is particularly valuable, when- ever the minimisation of software defects is an important issue.

This article is organised as follows: Section 2 describes the general architecture of the KeY system. Different scenarios of applying the system are discussed in Sect. 3. In Sect. 4 we introduce an example that is used throughout the rest of this paper. Section 5 describes the KeYspecific embedding of formal specifications into a UMLbased design process. Then, the formal analysis of the relationship of such specifications to each other (Sect. 6), as well as to a given implementation (Sect. 7), is discussed. Section 8 then describes how the resulting proof obligations are processed by interactive and automated theorem proving. After a brief look on implementation issues (Sect. 9), we describe some case studies performed with $\mathrm{KeY}$ (Sect. 10). Finally, we summarise the current state of the KeY project (Sect. 11), and in Sect. 12 draw some conclusions. The paper is an updated, extended, and completely rewritten version of $[2,3]$.

\section{Architecture of the $\mathrm{KeY}$ tool}

The KeY system is built on top of a commercial CASE tool. Integrating our system into an already existing tool has obvious advantages:

1. All features of the existing tool can be used and do not need to be reimplemented.

2. The software developer does not have to become familiar with a new design and development tool. Furthermore the developer is not required to change tools during development, everything that is needed is integrated into one tool.

A CASE tool that is well suited for our purposes has to be easily extensible and the extensions have to fit nicely into the tool providing a uniform user interface. We decided to use Together Control Center from Borland [23], in the following referred to as TogetherCC. Among all the tools on the market this one seems to be most suitable for our purposes. It has state-of-the-art development and UML support (including some very basic support for textual specifications) and can be extended in almost any possible way by JAVA modules - TogetherCC offers access to most of its "internals" by means of a JAVA open API (see Sect. 9). There is however no fundamental obstacle to adding the KeY extensions to other, similar CASE tools. Figure 1 shows a screenshot of TogetherCC with KeY system extensions.

The architecture of the KeY system is shown in Fig. 2. In the following, we briefly describe the components and the interactions between them:

1. The modelling component (upper part in Fig. 2) consists of the CASE tool with extensions for formal specification. While the CASE tool already allows the software model to contain OCL specifications, it does not have any support to create or process them in 


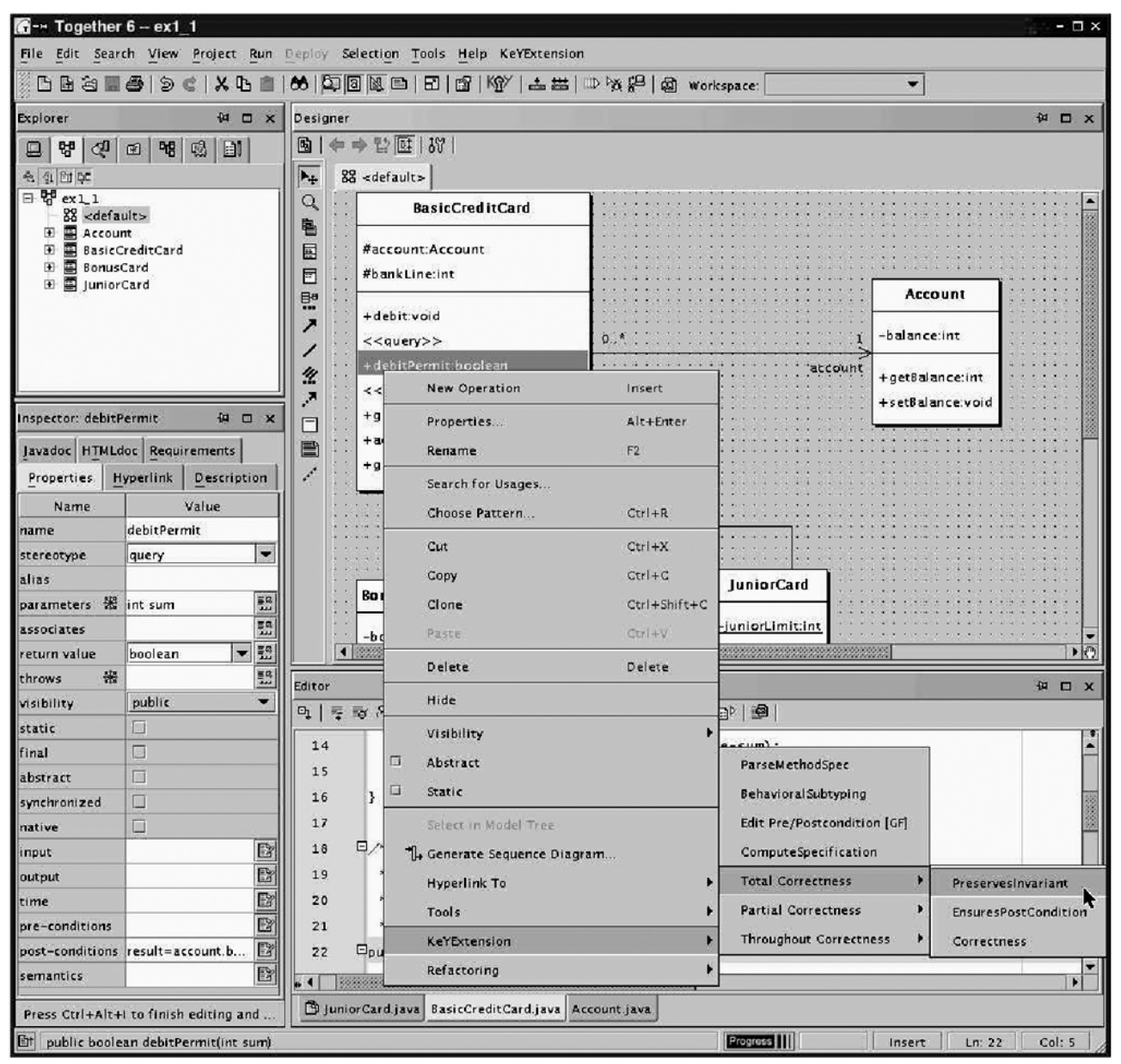

Fig. 1. TogetherCC with $\mathrm{KeY}$ system extensions

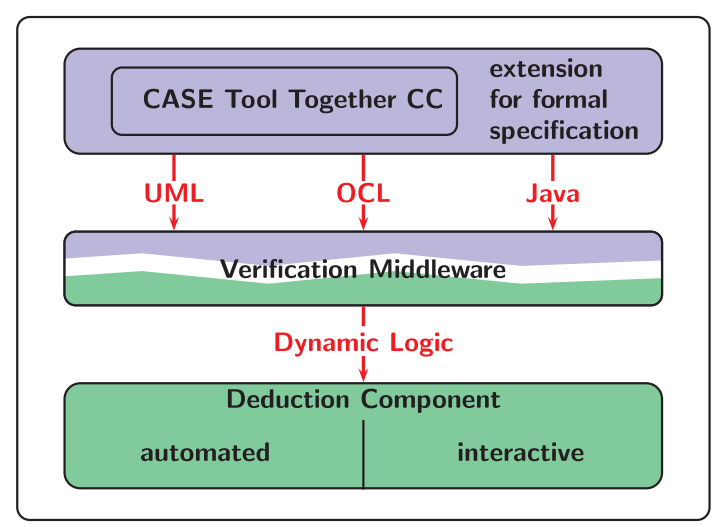

Fig. 2. The architecture of the KeY system

a formal way - OCL specifications are just textual annotations and are handled in the same way as comments. This is where the extension comes into play. It allows the user to create, process and prepare the OCL specifications (together with the model and its implementation) which can be later processed and passed to the deduction component. Manipulating OCL specifications is done by employing external programs and libraries $[31,33,41]$ as well as using TogetherCC's pattern mechanism to instantiate specifications from OCL specification templates [12] (see also Sect. 5). The CASE tool itself provides all the functionality for UML modelling and project development and is responsible for most of the user interactions with the project.

2. The verification middleware is the link between the modelling and the deduction component. It translates the model (UML), the implementation (JAVA) and the specification (OCL) into JAVA CARD Dynamic Logic proof obligations which are passed to the deduction component. JAVA CARD Dynamic Logic is a program logic used by the KeY prover (deduction component), see Sect. 7. The verification component is also responsible for storing and managing proofs during the development process.

3. The deduction component is used to construct proofs for JAVA CARD Dynamic Logic proof obligations generated by the verification component. It is an interactive verification system combined with powerful automated deduction techniques. All those components are fully integrated and work on the same data structures. 
All components are implemented in JAVA and fully integrated with TogetherCC through its open API resulting in a uniform user interface. In addition, some components of the $\mathrm{KeY}$ tool can be used stand alone: the OCL to JAVA CARD Dynamic Logic translator and the prover, see Sect. 9. Uniform implementation in JAVA makes the KeY tool portable. It should also be mentioned that all $\mathrm{KeY}$ system extensions can optionally be switched on and off in TogetherCC and thus it is the developer's decision to use them or not.

\section{$3 \mathrm{KeY}$ tool application scenarios}

The KeY tool can be used in various scenarios by people who have widely differing skills with formal methods. In the present section we sketch three main scenarios for the $\mathrm{KeY}$ tool: $\mathrm{KeY}$ in the development process of industrial software without particular demands on security, $\mathrm{KeY}$ in the development of security critical software, and, finally, $\mathrm{KeY}$ in education and training. Figure 3 shows the appropriate level of skill with formal methods for each environment.

As stated in the introduction, the aim of the $\mathrm{KeY}$ project is the integration of formal methods into the industrial software development process. Therefore, the most important target user group for the $\mathrm{KeY}$ tool are people who are not experts in formal methods. In many cases, users will even have reservations against formal methods.

As a consequence, to reach the goal of the project it is of crucial importance that the $\mathrm{KeY}$ tool allows for gradual verification, so that software engineers on any (including low) experience level with formal methods may benefit. In particular, the existence of full formal specification is not a prerequisite to make productive use of the $\mathrm{KeY}$ tool. The software engineer is free to determine the amount of formal methods he or she is willing to utilise.

The main use of $\mathrm{KeY}$ in an industrial environment is not necessarily full formal verification, but formal modelling. While implementations undergo frequent alterations and warrant formal verification only in exceptional cases, specifications are much less prone to changes. The

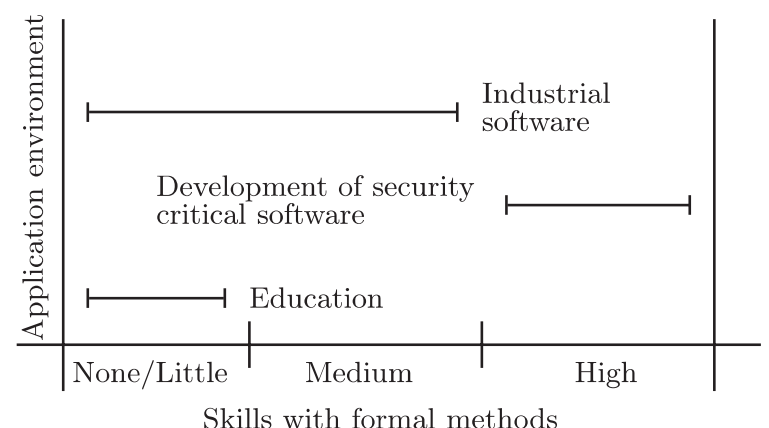

Fig. 3. KeY application environments and corresponding skill levels benefits of formal and, hence, unambigous specifications, are obvious. Moreover, our experience shows that many bugs are contained in specifications, and mere formalisation exhibits many of them [26].

To motivate users with few skills in formal methods or who have reservations against them, the $\mathrm{KeY}$ tool provides automatic support for creating formal specifications in several ways (see Sect. 5). For example, templates for often-needed OCL constraints are provided which we call $\mathrm{KeY}$ idioms. These KeY idioms can easily be instantiated by the user and the corresponding OCL constraints are then generated automatically. In addition, instantiating $\mathrm{KeY}$ patterns $[12,26]$, extensions of certain well-known design patterns, is another possibility to obtain a specification without having to know OCL syntax. Once an OCL-based formal specification is obtained, one could even hook up to other theorem provers that support a formal OCL semantics [25]. Finally, an authoring tool for OCL constraints [41] offers assistance in generating specifications and helps to understand OCL constraints by rendering them automatically in natural language. It is currently being integrated into the $\mathrm{KeY}$ tool. We believe that the user support provided by the KeY tool can help to overcome reservations against formal methods and, hopefully, increases the willingness of developers to give formal methods a try.

A second possible field of application for the $\mathrm{KeY}$ tool is the development (including formal verification) of security critical software [56]. Here, the high risks that emanate from faulty implementations warrant the effort of formal verification. An interesting possibility is the provision of a formally verified reference implementation. We stress that the KeY tool cannot merely be used for functional verification, but is also very suitable for formal analysis of security properties [30] (see also Sect. 10.4).

We do not claim that full formal software verification is possible without any skills in formal methods, so this application scenario pertains to formal methods experts. Since the KeY tool is an integrated system with a uniform user interface for modelling, specification, implementation, and verification of software, it can be used for the whole development process. This is an advantage of the $\mathrm{KeY}$ tool over conventional verification tools (for example, $[13,59,60]$ ). Without integration, several tools with typically incompatible interfaces have to be deployed to cover all steps from design to verification.

Another advantage of an integrated tool is that it enables efficient cooperation between developers whose skills in formal methods differs significantly (between none and high). This is important to make efficient use of those members of a development team that have training in formal methods.

The final, but no less important, scenario we mention is the use of $\mathrm{KeY}$ in education and training. Its modular architecture allows certain components to be used standalone which is of advantage here. 
The deduction component, for example, may be used stand-alone for teaching interactive theorem proving in first-order predicate logic or program logic. The authoring tool for OCL constraints and the OCL syntax checker are predestined to support teaching how to write formal specifications. But also the integrated tool can be used in a formal methods course. This has the fortunate effect to emphasise that formal software development can be complementary rather than alien to conventional methods. The KeY tool has been used successfully in various courses at Chalmers University, University of Karlsruhe, and University of Koblenz since 2002. We plan to publish a teaching unit for undergraduate level formal methods courses based on the KeY tool. The stand-alone components are also suitable for self-study, in particular, the authoring tool for OCL constraints.

\section{Running example}

Throughout the paper we will use a running example, simple enough to concisely illustrate the $\mathrm{KeY}$ concepts and mechanisms. It consists of a credit card application, the class diagram of which is depicted in Fig. 4, in its simplest form. We assume some familiarity with reading UML class diagrams. As a quick introduction to UML we recommend [34]. The main feature of the diagram in Fig. 4 is the class BasicCreditCard. In the sequel we often use the abbreviation $B C C$ for the name of this class. The class offers the operation debit to charge a certain sum to the credit card. Successive debits are accumulated in the balance attribute of the class Account. The debit operation is only permitted as long as the credit limit bankLine is not exceeded. Further operations allow to query the attributes bankLine and account and permissibility of a debit operation. These operations do not modify the system state and are, therefore, labelled with the UML stereotype «query». To make things a little bit more interesting we have included in the model two subclasses JuniorCard, which will have a stricter credit limit, and BonusCard, in which the debit operation in addition to its usual function may increase the bonus points stored in the bonus attribute depending on the result of the operation bonus. The Account class is modelled only rudimentary. We do, e.g., not consider the transfer of money to the account to balance the accumulated debts, let's say, at the end of the month.

\section{Embedding formal specification into the design process}

\subsection{Process models}

In industrial contexts of software development it became popular to take advantage of mainly graphical modelling notations such as the UML. Modelling notations vary in many aspects and are tailored to special purposes. It turns out that software developers have difficulties in practical application of modelling notations even if developers understood what the notations mean and for which purposes they should be used. To overcome this problem it is seen as best practice to follow certain guidelines according to which notation should be applied by software developers in each phase of a project. Such guidelines are known as process models. Most of these (for example, Extreme Programming (XP) [14], Rational Unified Process (RUP) [46], Boehm's spiral model [22]) include the basic

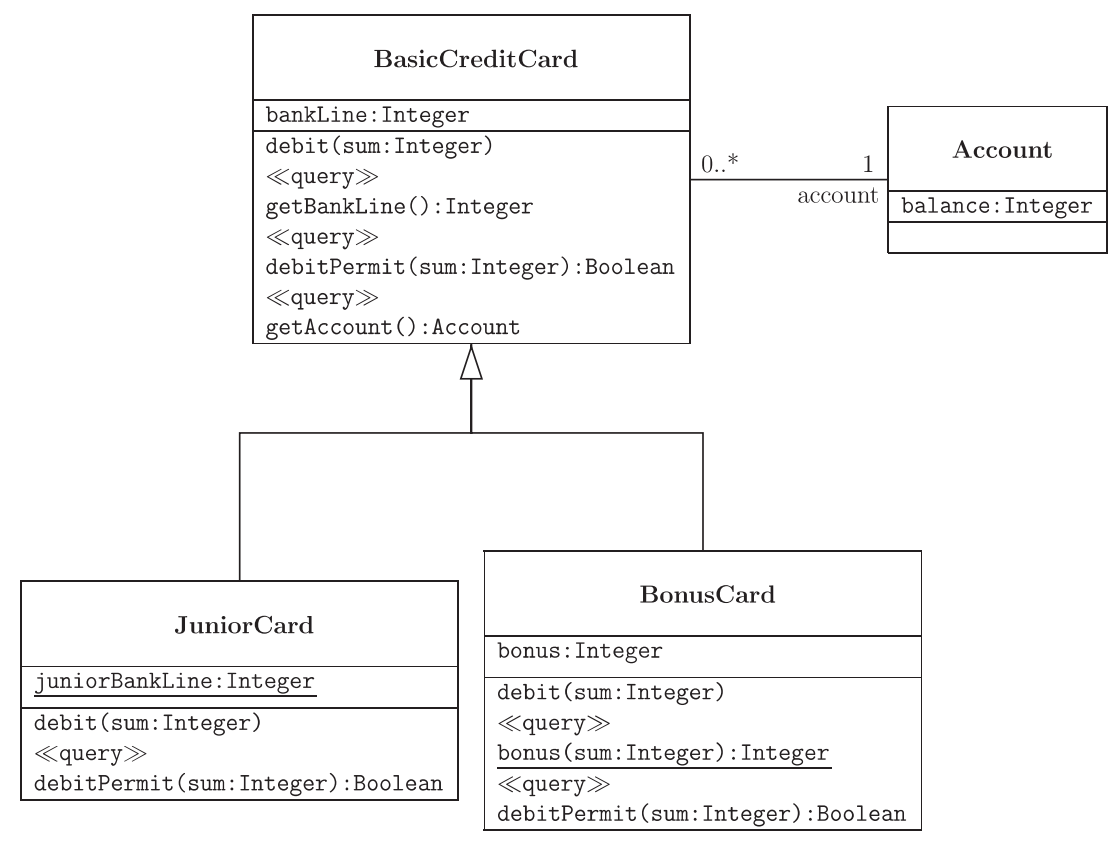

Fig. 4. A simple credit card scenario 
phases of software development: inception/analysis - design - implementation/test - deployment/maintenance. Modern process models tend to cycle through basic phases (iterative model) and to use at each development step all information available from artefacts created in previous development steps. The main concern of a process model is twofold:

1. To increase the productivity of the software developer.

2. To improve the quality of delivered software including the documentation so as to facilitate adaptations in the maintenance phase.

The main goal of the KeY project is the popularisation of formal methods in the industrial setting of software development. In a first step, formalisation is imported in the form of more precise models obtained through usage of the textual OCL. We focus on the application of OCL within class diagrams and we describe at which stage of a process model users can take advantage of OCL constraints.

\subsection{OCL constraints in the domain model}

The result of the inception/analysis phase is a domain model of the target system. The domain model ought to give an overview over concepts identified and the most important relationships among them. For the sake of flexibility and changeability in later phases, the domain model should not be too detailed. On the other hand, certain properties of domain classes become evident already in the first phase. As an example, the class diagram in Fig. 4 does not contain all the information that we want to be included in our model. The meaning of a bank (credit) line may be clear to a human reader, but it is not mirrored in our model so far and thus no analysis tool, that goes beyond syntactic checks, could make use of it. It is exactly for the purpose to express information of this kind that the OCL has been included in UML (see [72] for a quick introduction and [58] for the current language specification of OCL). OCL allows to add invariants such as

\section{context BasicCreditCard \\ inv: self . bankLine $>=0$ \\ inv: self . account. balance $>=-$ self . bankLine}

to the class BasicCreditCard. The intention is that the constraint should be satisfied in all system states, where the reserved variable self is implicitly quantified over all existing objects in the class BasicCreditCard.

Our experience with software developers working in an industrial context showed that they are often well aware of such constraints, which however are being documented in a rather informal way (if at all) so that no tool can make use of them [12]. Closer questioning reveals the reason: Software developers are not used to formulate constraints in a formal language such as OCL. The $\mathrm{KeY}$ tool offers a simple, but powerful mechanism to start authoring formal constraints in a gentle way. Users can generate formal constraints without the immediate need to learn specific syntax or keywords.

\section{$5.3 \mathrm{KeY}$ idioms}

The KeY tool contains a library of predefined constraints called $\mathrm{KeY}$ idioms. Users may choose an idiom from the library and instantiate it to the current target model by setting idiom-specific parameters. The desired constraint is then automatically generated according to the context of the target model.

The first of the invariants given above for class BasicCreditCard, for example, can be generated from an idiom. Calling the KeY idiom library for class BasicCreditCard results in the dialog displayed in Fig. 5. Filling in the values as shown, returns exactly the first invariant from above.

Not only invariants, but also pre- and postconditions can be generated in this way. These are attached to operations instead of classes.

The library of idioms is extensible by means of a simple scripting language. Hence, experienced users (or the formal methods expert in a development team) can write project-specific idioms. In addition, the generated OCL constraints can, of course, be manually changed afterwards. Even the generation of constraint skeletons may be useful in some situations.

What the KeY tool does not (yet) offer is a facility to "reverse engineer" OCL constraints, i.e., to find out which idiom a given constraint was generated from. Reverse engineering could provide a correspondence between possibly large and complex OCL constraints and abstract, descriptive, and more understandable idioms. There are two possibilities to attain similar goals. First, one may simply change idioms in such a way that suitable comments (like the name of the current idiom) are generated in addition to OCL constraints. The second possibility is the usage of an authoring tool for simultaneous development of natural language and OCL constraints [41]. It is currently being integrated into the $\mathrm{KeY}$ tool. With the help of this tool, the example above is thus rendered in English (German and Swedish are supported as well):

"The following invariant holds for all Basic-

CreditCards:

the bankLine of the BasicCreditCard is greater than or equal to zero."

It is possible to make the textual rendering ("linearisation") dependent of the type of an object. For example, one could write "bank line" instead of "bankLine" to enhance readability. While automatic translation from arbitrary natural language texts into OCL is unrealistic, the other direction is feasible. Even if the result is not always stylistically elegant, it is quite helpful, for example, to have an automatic rendering in English after making changes to the OCL. This opens up the possibility of "single source" technol- 


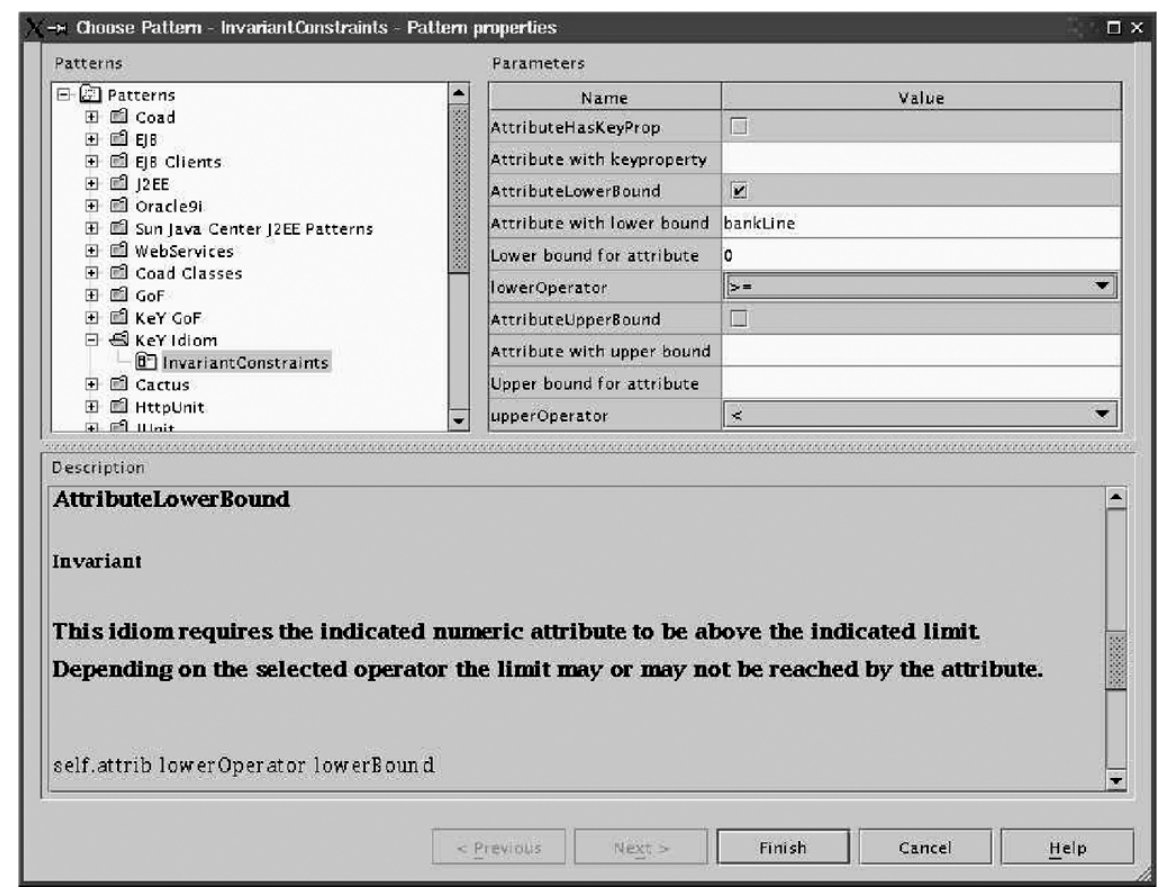

Fig. 5. Instantiation of $\mathrm{KeY}$ idiom AttributeLowerBound

ogy for informal and formal specifications. Without this, we foresee massive synchronisation and maintenance problems for formal specifications of non-trivial size.

$\mathrm{KeY}$ idioms help software developers to become faster acquainted with the syntax of a formal language. However, they provide only little help to decide which invariants and pre-/postconditions should be added to a model. It remains a task for the software developer to characterise the roles played by the classes in the domain model and the responsibilities they are assigned to. The $\mathrm{KeY}$ tool, as well as OCL, generally follow the designby-contract approach. We refer to [55, Chapter 11] for heuristics to find useful constraints.

\section{$5.4 \mathrm{KeY}$ patterns}

In the design phase, the domain model is transformed into a more detailed model in order to meet new requirements which were intentionally ignored in the first phase. For our running example in Fig. 4, such a requirement could be to change the kind of a credit card dynamically, e.g. a customer applies for the bonus program of the bank and hence his current credit card of type BasicCreditCard turns into a card of type BonusCard.

The transformation of a sparsely structured domain model into a more fine grained and appropriate model during the design phase is often facilitated by the application of design patterns. In the running example, the new requirements are best captured by application of the Decorator $[37$, pp. $175 \mathrm{ff}]$ pattern. In terms of the Decorator pattern, the type change from class BasicCreditCard to BonusCard for an object is seen as attaching additional responsibilities to this object. Technically, this is done by wrapping it in an object of type CardDecorator. The revised model after applying the Decorator pattern is displayed in Fig. 6.

The case tool TogetherCC offers special support for pattern application. When applying a pattern in TogetherCC, the user must indicate the roles of existing classes within the applied pattern. For example, the class $B a$ sicCreditCard is assigned to role concrete component and the class BonusCard to role concrete decorator. Based on these assignments, TogetherCC automatically generates further classes and even parts of the implementation according to the pattern definition. In the example, the class CardDecorator and the implementation of its method getBankLine are generated:

$$
\begin{aligned}
& \text { public int getBankLine }()\{ \\
& \quad \text { return component.getBankLine(); }
\end{aligned}
$$

In the $\mathrm{KeY}$ tool, the idea of pattern-application support was extended. $\mathrm{KeY}$ patterns are based on the well-known GoF patterns [37], but they contain constraints written in OCL that formally characterise important aspects of application scenarios. KeY patterns are instantiated in the same way as other patterns in TogetherCC but the user selects in addition appropriate textual constraints which are instantiated ${ }^{1}$ as well [12].

Like in the case of KeY idioms, the library of $\mathrm{KeY}$ patterns can be extended by the user. The predefined

1 The idea of instantiating textual constraints goes back at least to Syntropy [28] and the technique is used successfully in other contexts. One example is the proposed language description of UML 2.0 given in [54] where the process of instantiating constraints is called stamp out mechanism. 


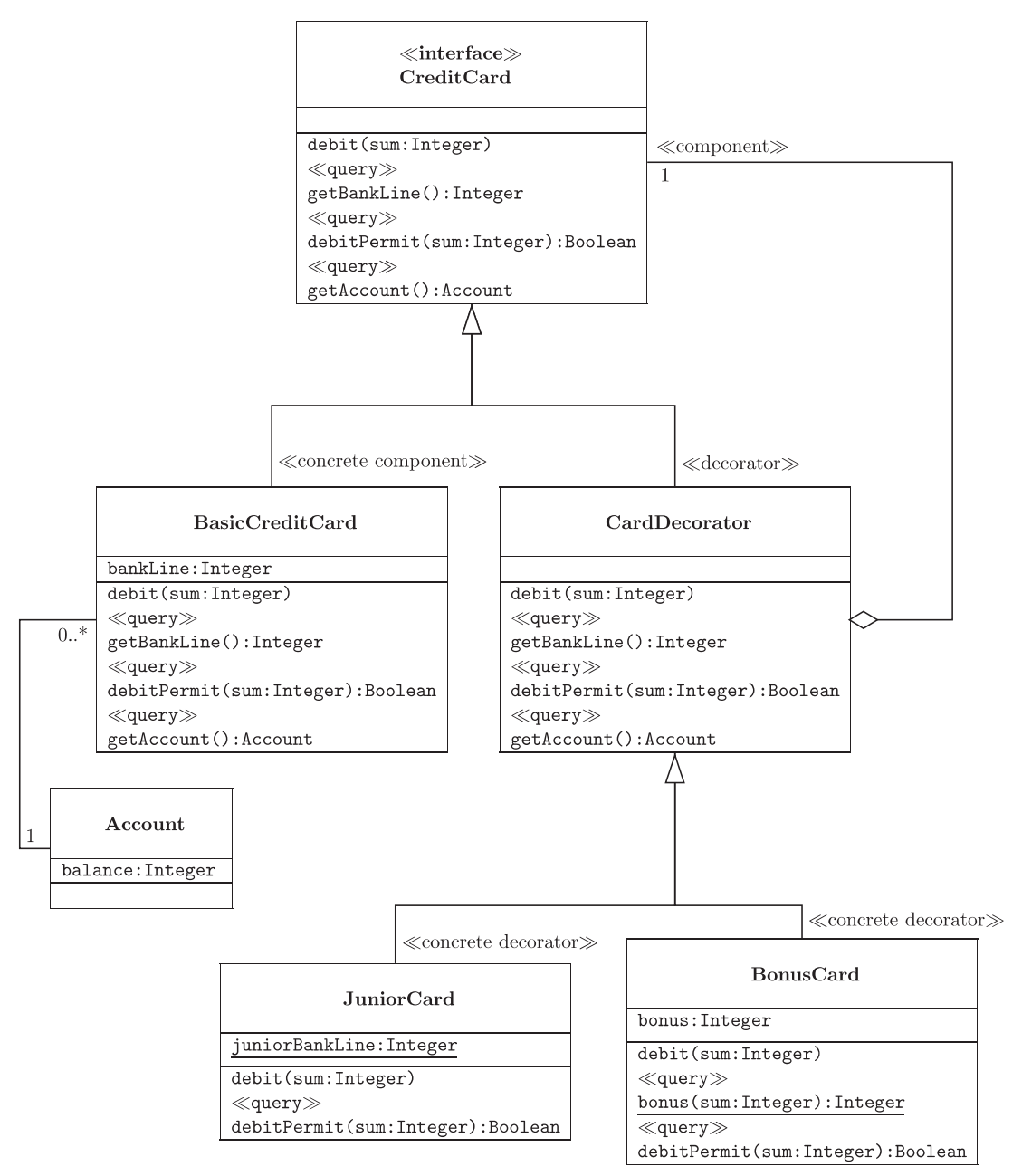

Fig. 6. Credit card diagram with Decorator pattern

version of the KeY Decorator pattern supports the generation of formal constraints for rather complex properties such as "no object of BonusCard has an inner component that has the type BonusCard". In terms of the Decorator pattern, this means that responsibilities can be attached to an object at most once. Here is a simpler example for a generated constraint: a postcondition of the operation CardDecorator: : getBankLine () to ensure that the implementation and specification of this operation generated during the application of the KeY pattern match each other:

context CardDecorator: : getBankLine() : Integer

post: result $=$ self . component $\cdot$ getBankLine ()

The instantiation of KeY patterns facilitates the creation of a specification in two ways. As in case of idioms, the difficulties of using a formal language like OCL are hidden from the user. Even more important, however, is the support in obtaining a complete specification: KeY patterns extend well-known GoF patterns for many scenarios. Listing all the predefined constraints that are useful in a given context reminds the user of aspects that might have been forgotten in the specification so far. In some cases the constraints attached to a pattern might contradict each other, so that choosing all of them would result in an inconsistent design. The KeY tool does not automatically detect such clashes between chosen constraints. However, it provides possibilities for computer-assisted analysis of the resulting constraints (see Sect. 6).

\section{Analysing specifications}

In this section we look at verification tasks that can be performed on the specification alone without reference to a possible or existing implementation. This is sometimes called horizontal verification. In the first subsection we describe what tasks are currently supported and in the following subsection we outline how these tasks are dealt with in the KeY system.

\subsection{Proof obligations}

Formal modelling as it is supported by the $\mathrm{KeY}$ system is based on OCL constraints which allow to characterise precisely the relationship between classes, attributes, and 
operations. A very simple example for an OCL constraint was already given by the invariants in Sect. 5.2. Now, we proceed on discussing further examples and give an overview to other kinds of constraints expressible in OCL and the most important subtleties of the language OCL. All constraints refer to our running example in Fig. 4.

The subclass JuniorCard of BasicCreditCard contains the class attribute juniorBankLine, i.e., the scope of this attribute is not individual objects but the whole class. The requirements we want this attribute to fulfil are described by these invariants:

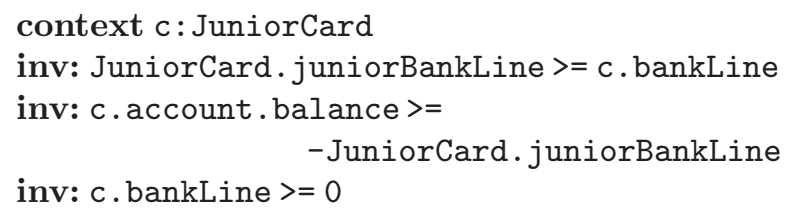

For every junior card $c$ the value of its bankLine attribute does not exceed the integer juniorBankLine, it is nonnegative, and the account of card c should not drop below - juniorBankLine.

Note that we take advantage of declaring a local variable $c$ and use it instead of self, which results in a more readable OCL constraint. Besides invariants, OCL also allows to add pre- and postconditions to operations.

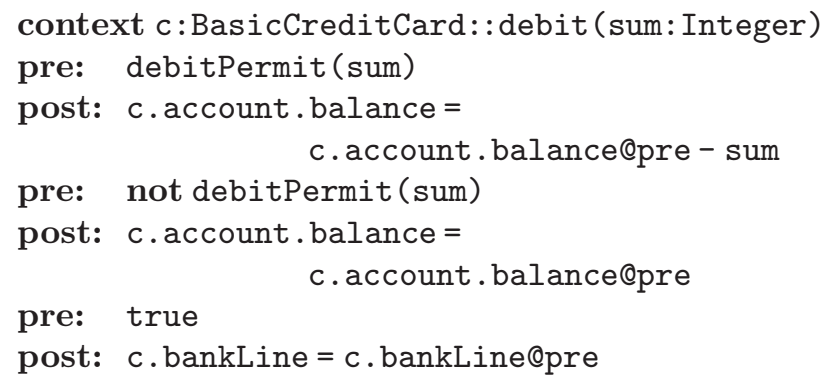

The OCL construct Qpre is only applicable in postconditions and causes the feature it decorates to refer to its value before the start of the operation. It is admissible to use the operation debitPermit in the constraints, because it has been specified as a query in the class diagram (Fig. 4).

The OCL language definition is not quite precise regarding the meaning of multiple pre-/postcondition pairs. We use a constraint with $n$ pre-/postcondition pairs as a convenient shorthand for $n$ constraints with respectively one pair each. Furthermore, multiple pre/postcondition pairs can be equivalently translated into a constraint with just one postcondition and precondition true. For the constraint above the following translation could be used:

context $c$ :BasicCreditCard::debit (sum: Integer)

pre: true

post: c.bankLine = c.bankLine@pre and if debitPermit@pre (sum)

then $c$.account. balance =

c. account.balance@pre-sum

else c.account. balance $=$

c. account.balance@pre
The OCL offers the predefined variable result to refer to the possible return value of an operation. This is particularly useful for query operations which are fully specified by fixing their return value.

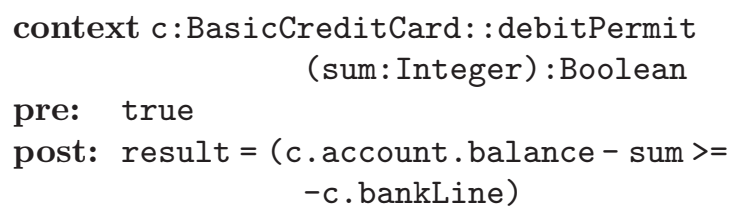

Note that we need not use the epre suffix for attributes in this statement, since we know by the query property of debitPermit that pre and post values coincide.

Pre- and postconditions are viewed, as is usual in the design-by-contract paradigm [55, Chapter 11], as two parts of a contract. If the client calling an operation makes sure that its precondition is satisfied, then the supplier of the operation guarantees that it terminates, and upon termination its postcondition holds.

Once a class diagram is supplemented with OCL invariants, pre- and postconditions, it is useful to analyse mutual dependencies among them. The simplest requirement, called structural subtyping, is to check whether the conjunction of all invariants of a subclass implies all invariants of its superclass. A quick glance at the above invariants shows that this is true for the subclass JuniorCard of BasicCreditCard (the invariants of the latter were given in Sect. 5.2). It is frequently assumed that an invariant of a subclass is an increment over the invariant of the superclass, i.e. the invariant in force for the subclass is the stated invariant plus the invariant of the superclass as an implicit conjunct. In this case structural subtyping would trivially be true. However, the incremental reading of invariants does not seem to be a universally accepted, so we offer support for the more liberal case.

A design methodology might require that operations preserve invariants, i.e., that for every operation op of class $C$ the precondition of op together with the invariant of $C$ logically implies the invariant in the successor state. If there is more than one precondition/postcondition pair to an operation this implication has, of course, to be proved for every pair. The operation debit in class BasicCreditCard does indeed preserve the invariant c. account.balance $>=-$ bankLine. The computation establishing this is straightforward and done fully automatically with the KeY tool.

Returning to Fig. 4, the debit operation in subclass BonusCard has additional functionality - it is supposed to increase the number of bonus points by bonus (sum) yielding the constraint:

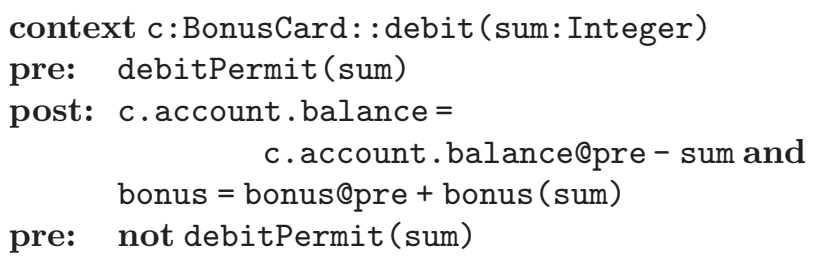




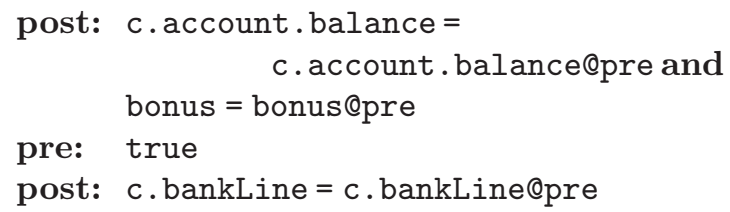

This allows us to illustrate another condition required by some design methodologies, called behavioural subtyping, or sometimes also the Liskov principle. It applies when an operation occurs in a class $_{1}$ with precondition pre $_{1}$ and postcondition post $t_{1}$ as well as in a subclass class $_{2}$ of class $_{1}$ with precondition pre $_{2}$ and postcondition post $t_{2}$.

Behavioural subtyping requires that the implications pre $_{1} \rightarrow$ pre $_{2}$ and post $_{2} \rightarrow$ post $_{1}$ be logically valid. These requirements can be justified when one accepts that the subclass relation entails: any object of class $_{2}$ can be used in any circumstances that an object from class $_{1}$ could be used. It is a trivial observation that the behavioural subtyping regime holds true for BasicCreditCard and its subclass BonusCard with respect to the operation debit. In case of multiple pre-/postcondition pairs it is best to equivalently translate them into a constraint with just one postcondition and one precondition, as mentioned above.

Both behavioural and structural subtyping, as well as the preservation of invariants, are supported by the $\mathrm{KeY}$ tool.

\subsection{Proving obligations}

When the user selects either one of the subtyping tasks or an invariant preservation task from the $\mathrm{KeY}$ extension menu within TogetherCC, a verification condition, formalised in dynamic logic, is generated and passed on to KeY's deduction system. To this end, the information contained in the UML class diagram as well as the OCL constraints have to be translated into dynamic logic.

This translation fixes a particular semantics for UML/ OCL. Quite a number of papers $([24,32,35]$ to name just a few) have been published doing the same, fixing a formal semantics by translating UML diagrams into some known formal system. Despite the often voiced need of a precise semantics for UML the informal semantics description did not lead to major discrepancies (at least for class diagrams and not touching issues of the metamodel). For OCL the situation was a less satisfactory. Most of the trouble arose from its meta-model and the integration into the rest of UML. These issues have been rigorously resolved in the submission [57] for the UML2.0 which is awaiting approval. Its formal semantics is based on the $\mathrm{PhD}$ thesis [63].

In the following we describe our translation from UML class diagrams with OCL constraints into typed dynamic logic by way of example. A full account can be found in the paper [49]. Summaries of parts of it were published as $[17,65]$.

The first step in the translation is to fix the vocabulary to be used on the logical side. This is straight forward: for
Types:

\begin{tabular}{|c|c|}
\hline category & names \\
\hline \hline model types & $B C C$, JuniorCard, BonusCard, Account \\
\hline OCL basic types & Integer, Boolean,.. \\
\hline OCL collection types & Set $_{B C C}$, Sequence \\
\hline
\end{tabular}

Functions:

\begin{tabular}{|c|c|}
\hline name & signature \\
\hline \hline bankLine & $B C C \rightarrow$ Integer \\
\hline balance & Account $\rightarrow$ Integer \\
\hline bonus & BonusCard $\rightarrow$ Integer \\
\hline juniorBankLine & Integer \\
\hline account & $B C C \rightarrow$ Account \\
\hline bcc & Account $\rightarrow$ Set \\
BCC \\
\hline debitPermit & $B C C \times$ Integer $\rightarrow$ Boolean \\
\hline$\vdots$ & \\
\hline
\end{tabular}

Fig. 7. Vocabulary for the simple credit card scenario in Fig. 4

every class in the UML model there will be a type in the logic, built-in OCL types are mapped onto corresponding abstract data types. Attributes, associations and query operations are mapped into functions in the obvious manner. Class attributes (e.g., juniorBankLine) turn into constants. We gloss over some details like naming and disambiguating conventions, except for the remark that unlabelled association ends get by default the name of the class they are attached to (e.g., bcc: Account $\rightarrow S t_{B C C}$ ). A selection of the vocabulary for the class diagram in Fig. 4 is shown in Fig. 7. The second invariant in Sect. 5.2 reads in logic as follows:

$\forall x: B C C .(x . a c c o u n t . b a l a n c e \geq-x$. bankLine $)$

We decided to stick also on the logical side with the dot notation as opposed to the traditional notation using brackets, in which the above formula would read $\forall x: B C C$. (balance $(\operatorname{account}(x)) \geq-$ bankLine $(x))$. This way it is possible to keep track of OCL constraints even when using the interactive theorem prover, see Fig. 8. To establish the structural subtyping property for the subclass JuniorCard of BasicCreditCard the following formula has to be proved to be a tautology:

$\forall c$ : JuniorCard.

$$
\begin{aligned}
& ((\text { juniorBankLine } \geq \text { c.bankLine } \wedge \\
& \text { c.account.balance } \geq- \text { juniorBankLine } \wedge \\
& \text { c.bankLine } \geq 0) \\
& \rightarrow \\
& (\text { c.bankLine } \geq 0 \wedge \\
& \text { c.account.balance } \geq- \text { c.bankLine }))
\end{aligned}
$$

Let us look at a new invariant for the class Account in Fig. 4:

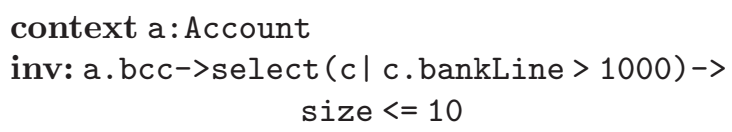

The same account may be used by different credit cards. The constraint says that at most 10 credit cards with 


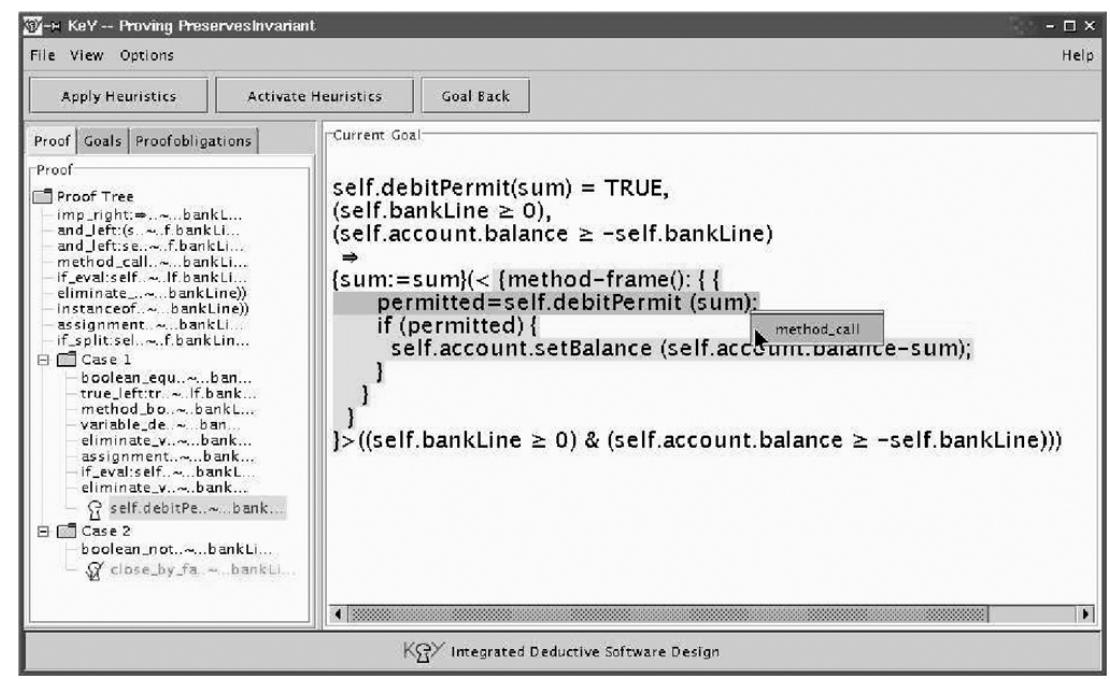

Fig. 8. The KeY prover window

credit limit exceeding 1000 can share the same account. Its translation into first-order logic reads

$\forall a:$ Account. $\left(\right.$ a.bcc.select ${ }_{E}$. size $\left.\leq 10\right)$

select $_{E}: \operatorname{Set}_{B C C} \rightarrow \operatorname{Set}_{B C C}$ is a new function symbol depending on the expression $E=\operatorname{select}(c \mid$ c.bankLine $>$ 1000). If we use $\emptyset$ (of type $S_{B C C}$ ) and insert (of type $\left.B C C \times S e t_{B C C} \rightarrow S e t_{B C C}\right)$ as the constructors of the abstract data type $\operatorname{Set}_{B C C}$ then the definition of select $_{E}$ reads

$\emptyset$ select $_{E}=\emptyset$

c.bankLine $>1000 \rightarrow c$.insert $(s)$. select $_{E}=$ c.insert $\left(\right.$ s.select $\left._{E}\right)$

c.bankLine $\leq 1000 \rightarrow c$.insert $(s)$. select $_{E}=$ s.select $_{E}$

All these formulas are passed on to the deduction system. The translation of the @pre construct requires more than first-order logic and will be explained in Sect. 7.3.

\section{Verifying correctness of implementations}

Besides supporting the analysis of a specification, $\mathrm{KeY}$ provides functionality for checking the correctness of a JAVA implementation with respect to a given UML/OCL specification.

In particular, $\mathrm{KeY}$ allows (1) to prove that after running a method, the method's post-condition holds, and (2) to prove that a method preserves a class invariant (program correctness requires that all public methods preserve all invariants).

\subsection{Dynamic logic}

We use an instance of dynamic logic (DL) $[42,43,51,61]$ - which can be seen as an extension of Hoare logic - as the logical basis of the KeY system's software verification component. Deduction in DL is based on symbolic program execution and simple program transformations and is, thus, close to a programmer's understanding of JAVA. DL is used in the software verification systems KIV [13] and VSE [45] for (artificial) imperative programming languages. More recently, the KIV system supports also a fragment of the JAVA language [67]. In both systems, DL was successfully applied to verify software systems of considerable size.

DL can be seen as a modal logic with a modality $\langle p\rangle$ for every $\operatorname{program} p$ (we allow $p$ to be any sequence of legal JAVA CARD statements); $\langle p\rangle$ refers to the successor worlds (called states in the DL framework) that are reachable by running the program $p$. In standard DL there can be several such states (worlds) because the programs can be non-deterministic; but here, since JAVA programs are deterministic, there is exactly one such world (if $p$ terminates) or there is no such world (if $p$ does not terminate). The formula $\langle p\rangle \phi$ expresses that the pro$\operatorname{gram} p$ terminates in a state in which $\phi$ holds. A formula $\phi \rightarrow\langle p\rangle \psi$ is valid if for every state $s$ satisfying precondition $\phi$ a run of the $\operatorname{program} p$ starting in $s$ terminates, and in the terminating state the post-condition $\psi$ holds.

Thus, the formula $\phi \rightarrow\langle p\rangle \psi$ is similar to the Hoare triple $\{\phi\} p\{\psi\}$. But in contrast to Hoare logic, the set of formulas of DL is closed under the usual logical operators: In Hoare logic, the formulas $\phi$ and $\psi$ are pure first-order formulas, whereas in DL they can contain programs. DL allows to involve programs in the descriptions $\phi$ resp. $\psi$ of states. For example, using a program, it is easy to specify that a data structure is not cyclic, which is impossible in pure first-order logic. Also, all JAVA constructs are available in our DL for the description of states (including while loops and recursion). It is, therefore, not necessary to define an abstract data type state and to represent states as terms of that type; instead DL formulas can be used to give a (partial) description of states, which 
is a more flexible technique and allows to concentrate on the relevant properties of a state.

\subsection{Syntax of JAVA CARD DL}

As said above, a dynamic logic is constructed by extending some non-dynamic logic with a modal operator $\langle\cdot\rangle$. In addition, we use the dual operator $[\cdot]$, for which $[p] \phi \equiv \neg\langle p\rangle \neg \phi$. The non-dynamic base logic of our DL is typed first-order predicate logic. We do not describe in detail what the types of our logic are (basically they are identical to the JAVA types) nor how exactly terms and formulas are built. The definitions can be found in [15]. Note that terms (which we often call "logical terms" in the following) are different from JAVA expressions; the former never have side effects.

In order to reduce the complexity of the programs occurring in formulas, we introduce the notion of a program context. The context can consist of any JAVA CARD program, i.e., it is a sequence of class and interface definitions. Syntax and semantics of JAVA CARD DL formulas are then defined with respect to a given context; and the programs in JAVA CARD DL formulas are assumed not to contain class definitions.

The programs in JAVA CARD DL formulas are basically executable statements of JAVA CARD code. The verification of a given program can be thought of as symbolic code execution. As will be detailed below, each rule of the calculus for JAVA CARD DL specifies how to execute one particular JAVA statement, possibly with additional restrictions. When a loop or a recursive method call is encountered, it is necessary to perform induction over a suitable data structure.

Given that we follow the symbolic execution paradigm for verification, it is evident that a certain amount of runtime infrastructure must be represented in JAVA CARD DL. It would be possible, but clumsy and inefficient, to achieve this by purely logical means. Therefore, we introduced an additional construct for handling of method calls that is not available in plain JAVA CARD. Methods are invoked by syntactically replacing the call by the method's implementation. To handle the return statement in the right way, it is necessary (a) to record the object field or variable $x$ that the result is to be assigned to, and (b) to mark the boundaries of the implementation body when it is substituted for the method call. For that purpose, we allow statements of the form method-frame $(x)\{$ body $\}$ to occur in JAVA CARD DL programs. Note, that this is a "harmless" extension because the additional construct is only used for proof purposes and never occurs in the verified JAVA CARD programs.

\subsection{Proof obligations}

Let us now turn to the translation of OCL constraints into JAVA CARD DL proof obligations. To prove that a method $\mathrm{m}\left(\arg _{1}, \ldots, \arg _{n}\right)$ of class $\mathrm{C}$ satisfies a pre/post-condition pair, the OCL conditions are first translated into first-order formulas pre (self, $\left.\arg _{1}, \ldots, \arg _{n}\right)$ and $\operatorname{post}\left(\operatorname{self}, \arg _{1}, \ldots, \arg _{n}\right.$ ), respectively (as described in Sect. 6). From these formulas, KeY constructs the JAVA CARD DL proof obligation

$$
\begin{aligned}
& \operatorname{pre}\left(\text { self, } \arg _{1}, \ldots, \arg _{n}\right) \rightarrow \\
& \quad\left\langle\text { self.m }\left(\arg _{1}, \ldots, \arg _{n}\right) ;\right\rangle \operatorname{post}\left(\text { self, } \arg _{1}, \ldots, \arg _{n}\right),
\end{aligned}
$$

where now self and $\arg _{1}, \ldots, \arg _{n}$ are program variables, which are implicitly universally quantified w.r.t. their initial value.

For example, the first pre-/postcondition pair for the debit operation from Sect. 6 is transformed into

$$
\begin{aligned}
& \text { c. debitPermit }(\text { sum })=\text { TRUE } \rightarrow \\
& \langle\text { c.debit(sum }) ;\rangle(\text { c.account.balance }= \\
& \text { c.account.balance@pre-sum }) .
\end{aligned}
$$

The call to the operation debit is translated into the JAVA CARD DL program "c.debit(sum);" that appears within angle brackets in the above formula. Furthermore balance@pre is a new function symbol with the same signature as balance. There are several possibilities to ensure that the function balance@pre has the intended semantics, see [10] for a detailed account. The simplest way is by adding a definition of balance@pre to the above formula:

$$
\begin{aligned}
& \forall x: \text { Account. }(x . \text { balance } @ \text { pre }=x . \text { balance } \wedge \\
& \text { c.debitPermit }(\text { sum })=\mathrm{TRUE} \rightarrow \\
& \quad\langle\text { c.debit(sum }) ;\rangle(\text { c.account.balance }= \\
& \quad \text { c.account.balance } @ \text { pre }- \text { sum })) .
\end{aligned}
$$

Similarly, to prove that a method $\mathrm{m}\left(\arg _{1}, \ldots, \arg _{n}\right)$ preserves an invariant, the proof obligation

$\left(i n v(\right.$ self $) \wedge$ pre $\left(\right.$ self $\left.\left., \arg _{1}, \ldots, \arg _{n}\right)\right) \rightarrow$

$\left\langle\right.$ self.m $\left.\left(\arg _{1}, \ldots, \arg _{n}\right) ;\right\rangle i n v$ (self)

is constructed, where inv(self) is the first-order translation of the invariant.

\subsection{Deductive calculus for proving obligations}

As usual for deductive program verification, we use a sequent-style calculus. A sequent is of the form $\Gamma \vdash \Delta$, where $\Gamma, \Delta$ are duplicate-free lists of formulas. Intuitively, its semantics is the same as that of the formula $\bigwedge \Gamma \rightarrow \bigvee \Delta$.

Rules of a sequent calculus are often represented by rule schemata, such as the example rules in the rest of this section. In the KeY system, rules are implemented using the taclet mechanism (see Sect. 8.1).

A proof for a goal (a sequent) $S$ is an upside-down tree with root $S$. In practice, rules are applied from bottom to top. That is, proof construction starts with the initial proof obligation at the bottom and ends with axioms (rules with an empty premiss tuple). 
Since our JAVA CARD DL calculus contains (at least) one rule for each JAVA CARD programming construct (there are about 250 rules for handling the JAVA part of the logic), we cannot present all rules in this paper. Instead we describe some important ones, which are exemplary for their respective class of rules.

\subsubsection{The active statement in a program}

The rules of our calculus operate on the first active command $p$ of a program $\pi p \omega$. The non-active prefix $\pi$ consists of an arbitrary sequence of opening braces " $\{$ ", labels, beginnings "try\{" of try-catch-finally blocks, and beginnings "method-frame $(. .).\{$ " of method invocation blocks. The prefix is needed to keep track of the blocks that the (first) active command is part of, such that the abruptly terminating statements throw, return, break, and continue can be handled appropriately. ${ }^{2}$ The postfix $\omega$ denotes the "rest" of the program, i.e., everything except the non-active prefix and the part of the program the rule operates on. For example, if a rule is applied to the following JAVA block operating on its first active command " $\mathrm{i}=0 ; "$, then the non-active prefix $\pi$ and the "rest" $\omega$ are the indicated parts of the block:

$\underbrace{\text { I: }\{\text { try }}_{\pi}\{\mathrm{i}=0 ; \underbrace{\mathrm{j}=0 ;\} \text { finally }\{\mathrm{k}=0 ;\}\}}_{\omega}$

\subsubsection{The assignment rule and handling state updates}

In JAVA (like in other object-oriented programming languages), different object variables can refer to the same object. This phenomenon, called aliasing, causes serious difficulties for handling of assignments in a calculus for JAVA CARD DL.

For example, whether or not a formula "o1.a =1" still holds after the (symbolic) execution of the assignment "o2. a = 2;", depends on whether or not o1 and o2 refer to the same object.

Therefore, JAVA assignments cannot be symbolically executed by syntactic substitution. Solving this problem naively - by doing a case split if the effect of an assignment is unclear - is inefficient and leads to heavy branching of the proof tree.

In our JAVA CARD DL calculus we use a different solution. It is based on the notion of updates. These (state) updates are of the form $\langle l o c:=v a l\rangle$ and can be put in front of any formula. The semantics of $\langle l o c:=v a l\rangle \phi$ is the same as that of $\langle l o c=v a l ;\rangle \phi$. The difference between an

\footnotetext{
${ }^{2}$ In DL versions for simple artificial programming languages, where no prefixes are needed, any formula of the form $\langle p q\rangle \phi$ can be replaced by $\langle p\rangle\langle q\rangle \phi$. In our calculus, splitting of $\langle\pi p q \omega\rangle \phi$ into $\langle\pi p\rangle\langle q \omega\rangle \phi$ is not possible (unless the prefix $\pi$ is empty) because $\pi p$ is not a valid program; and the formula $\langle\pi p \omega\rangle\langle\pi q \omega\rangle \phi$ cannot be used either because its semantics is in general different from that of $\langle\pi p q \omega\rangle \phi$.
}

update and an assignment is syntactical. The expressions $l o c$ and val must be simple in the following sense: loc is (a) a program variable var, or (b) a field access obj.attr, or (c) an array access arr[i]; and val is a logical term (that is free of side effects). More complex expressions are not allowed in updates.

The syntactical simplicity of $l o c$ and $v a l$ has semantical consequences. In particular, computing the value of $v a l$ has no side effects. The KeY system uses special simplification rules to compute the result of applying an update to logical terms and formulas not containing programs. Computing the effect of an update to a program $p$ (and a formula $\langle p\rangle \phi$ ) is delayed until $p$ has been symbolically executed using other rules of the calculus. Thus, case distinctions are not only delayed but they can often be avoided completely, because (a) updates can be simplified before their effect is computed and (b) their effect is computed when a maximal amount of information is available (namely after the symbolic execution of the program).

The assignment rule now takes the following form $(\mathcal{U}$ stands for an arbitrary sequence of updates):

$\frac{\Gamma \vdash \mathcal{U}\langle l o c:=v a l\rangle\langle\pi \omega\rangle \phi}{\Gamma \vdash \mathcal{U}\langle\pi l o c=v a l ; \omega\rangle \phi}$

That is, it just adds the assignment to the list of updates $\mathcal{U}$. Of course, this does not solve the problem of computing the effect of the assignment. This problem is postponed and solved by rules for simplifying updates.

This assignment rule can, of course, only be used if the expression val is a logical term. Otherwise, other rules have to be applied first to evaluate val (as that evaluation may have side effects). For example, these rules replace the formula $\langle\mathrm{x}=++\mathrm{i} ;\rangle \phi$ with $\langle\mathrm{i}=\mathrm{i}+1 ; \mathrm{x}=\mathrm{i} ;\rangle$. One can view these rules as on-the-fly program transformations. Their effect is always local and fairly obvious, so that the user's understanding of the proof is not obfuscated.

\subsubsection{The rule for if-else}

As a first example for a rule with more than one premiss, we present the rule for the if statement.

$\Gamma, \mathcal{U}(b=$ TRUE $) \vdash \mathcal{U}\langle\pi p \omega\rangle \phi$
$\frac{\Gamma, \mathcal{U}(b=\text { FALSE }) \vdash \mathcal{U}\langle\pi q \omega\rangle \phi}{\Gamma \vdash \mathcal{U}\langle\pi \text { if }(b) p \text { else } q \omega\rangle \phi}$

The two premisses of this rule correspond to the two cases of the if statement. The semantics of rules is that, if all the premisses are true in a state, then the conclusion is true in that state. In particular, if the premisses are valid, then the conclusion is valid.

As the if rule demonstrates, applying a rule (from bottom to top) corresponds to a symbolic execution of the program to be verified. 


\subsubsection{The rule for while loops}

The following rule "unwinds" while loops. Its application is the prerequisite for symbolically executing the loop body. These "unwind" rules allow to handle while loops if used together with induction schemata for primitive and user defined data types.

$\frac{\Gamma \vdash\left(\left\langle\pi \text { if }(c) l^{\prime}:\left\{l^{\prime \prime}:\left\{p^{\prime}\right\} l_{1}: \cdots l_{n}: \text { while }(c)\{p\}\right\} \omega\right\rangle \phi\right)}{\Gamma \vdash\left(\left\langle\pi l_{1}: \cdots l_{n}: \text { while }(c)\{p\} \omega\right\rangle \phi\right)}$

where

- $l^{\prime}$ and $l^{\prime \prime}$ are new labels,

- $p^{\prime}$ is the result of (simultaneously) replacing in $p$

(a) every break $l_{i}$ (for $1 \leq i \leq n$ ) and every break (with no label) that has the while loop as its target by break $l^{\prime}$, and

(b) every continue $l_{i}$ (for $1 \leq i \leq n$ ) and every continue (with no label) that has the while loop as its target by break $l^{\prime \prime} .^{3}$

The list " $l_{1}: \cdots l_{n}:$ " usually is empty or has only one element, but in general a loop can have more than one label.

In the "unwound" instance $p^{\prime}$ of the loop body $p$, the label $l^{\prime}$ is the new target for break statements and $l^{\prime \prime}$ is the new target for continue statements, which both had the while loop as target before. This results in the desired behaviour: break abruptly terminates the whole loop, while continue abruptly terminates the current instance of the loop body.

A continue with or without label is never handled by a rule directly, because it can only occur in loops, where it is always transformed into a break by the loop rules.

\subsubsection{The rules for try/throw}

The following rules allow to handle try-catch-finally blocks and the throw statement. These are simplified versions of the actual rules that apply to the case where there is exactly one catch clause and one finally clause.

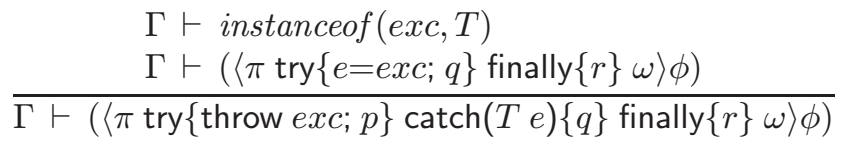

$\Gamma \vdash \neg$ instanceof $($ exc,$T) \quad \Gamma \vdash(\langle\pi r$; throw exc; $\omega\rangle \phi)$ $\overline{\Gamma \vdash(\langle\pi \text { try }\{\text { throw } e x c ; p\} \operatorname{catch}(T e)\{q\} \text { finally }\{r\} \omega\rangle \phi)}$

The predicate instanceof $(e x c, T)$ has the same semantics as the instanceof operator in JAVA. It evaluates to true if the value of exc is assignable to a program variable of type $T$, i.e., if its dynamic type is a sub-type of $T$.

3 The target of a break or continue statement with no label is the loop that immediately encloses it.
Rule (4) applies if an exception exc is thrown that is an instance of exception class $T$, i.e., the exception is caught; otherwise, if the exception is not caught, rule (5) applies.

\section{Interactive and automated proof construction}

\subsection{Taclets}

Most existing interactive theorem provers are "tactical theorem provers". The tactics for which these systems are named are programs which act on the proof tree, mostly by many applications of primitive rules, of which there is a small, fixed set. The user constructs the proof by selecting the tactics to run. Writing a new tactic for a certain purpose, e.g. to support a new data type theory requires expert knowledge of the theorem prover.

In the KeY prover, both tactics and primitive rules are replaced by the taclet concept $[16,40]$. A taclet combines the logical content of a sequent calculus rule with pragmatic information that indicates when and for what it should be used. In contrast to the usual fixed set of primitive rules, taclets can easily be added to the system. They are formulated as simple pattern matching and replacement schemas. For instance, a typical taclet might read as follows:

find $(b->c==>)$ if $(b==>)$ replacewith

(c $==>$ ) heuristics(simplify)

This means that an implication $b->c$ on the left side of a sequent may be replaced by $c$, if the formula $b$ also appears on the left side of that sequent. Apart from this "logical" content, the keyword find indicates that the taclet will be attached to the implication and not to the formula $b$ for interactive selection, see Sect. 8.3. The clause heuristics(simplify) indicates that this rule should be part of the heuristic named simplify, meaning that it should be applied automatically whenever possible if that heuristic is activated, see Sect. 8.4.

While taclets can be more complex than the typically minimalistic primitive rules of tactical theorem provers, they do not constitute a tactical programming language. There are no conditional statements, no procedure calls and no loop constructs. This makes taclets easier to understand and easier to formulate than tactics. In conjunction with an appropriate mechanism for application of heuristics, they are nevertheless powerful enough to permit interactive theorem proving in a convenient and efficient way [40].

In principle, nothing prevents one from formulating a taclet that represents an unsound proof step. It is possible, however, to generate a first-order proof obligation from a taclet, at least for taclets not involving programs. If that formula can be proven using a restricted set of "primitive" taclets, then the new taclet is guaranteed to 
be a correct derived rule. As for the primitive taclets for handling JAVA programs in JAVA CARD DL, it is possible to show their correctness using the Isabelle formalisation of JAVA by Oheimb [70,71].

\subsection{Proof visualisation}

The KeY prover window (see Fig. 8) consists of two panes, the left of which has three tabs. The tab called Proof contains a tree representing the current proof state. The nodes of the tree correspond to sequents (goals) at different proof stages. One can click on any node to see the corresponding sequent and the rule that was applied on it in the following proof step (except when the node is a leaf). Leaf nodes in open proof branches are coloured red, whereas leaves of closed branches are coloured green. The tab named Goals lists the open proof goals. By clicking on any goal, one can change the active goal that is displayed in the right pane. When an active goal is open, one can work towards its closure by applying proof rules interactively or by activating automated proof search. The $\mathrm{KeY}$ prover allows the user to work on several proof obligations simultaneously. The third tab, named Proof obligations, keeps track of all currently open proofs and lets the user switch between them. For more information on the user interface of the $\mathrm{KeY}$ prover, see also [39].

\subsection{Support for interactive proof construction}

Depending on where the mouse pointer is moved, one sub-formula or sub-term of the goal, the focus term, is highlighted (for example, in Fig. 8, the diamond operator and the program it contains is the focus term). More precisely, a term is put into focus by pointing at its top-level symbol. Then, pressing the left mouse button displays a list of all proof rules currently applicable to the focus term (in the example, only the method call rule is applicable). One of these can be selected and applied interactively, thus generating a new proof goal.

\subsection{Automated proof search}

Automated proof search is performed by applying "heuristics" which can be seen as a collection of rules suited for a certain task. For example, the heuristic simplify_boolean contains rules to simplify boolean expressions. The user can activate and de-activate heuristics depending on the state of the proof and goal he or she wants to tackle next. And the automatic application of heuristics can easily be switched on and off during proof construction. The prover can automatically find quantifier instantiations in a way similar to free variable tableaux. Backtracking in the proof search is avoided through the incremental closure technique of [38].

\section{Implementation issues}

From the user perspective, the $\mathrm{KeY}$ tool is an extension of the commercial case tool TogetherCC. The open API of TogetherCC allows the KeY tool to add items to the CASE tool's contextual menus for classes, methods, etc. The API also makes it possible to modify the currently open UML model, for instance, during pattern instantiation (see Sect. 5).

\subsection{Used technology}

The KeY tool is implemented in the JAVA programming language. This choice has several advantages, besides the obvious one of portability. Using the JAVA language makes it easy to link the $\mathrm{KeY}$ tool to TogetherCC, which is also written in JAVA. More generally, JAVA is well suited for interaction with other tools, written in JAVA or not. In particular, the imperative nature of the language leads to a comparatively natural native code interface, in contrast to the logic or functional programming languages often preferred for deduction purposes.

JAVA was also a good choice for the construction of the graphical user interface, which is an important aspect of the KeY tool. Finally, previous experiments with both interactive [40] and automated [38] theorem proving have shown that the advantages of JAVA outweigh the additional effort for the implementation of term data structures, unification, etc.

A simple form of parametric genericity is used in the implementation. For instance, instead of the usual interfaces Set and List, there are interfaces Listof Integer, Set0fTerm, etc.; they are semi-automatically generated from templates. This approach leads to a certain "code bloat", but it improves readability and type safety.

Apart from TogetherCC, the KeY tool makes use of various third party software. Parsers are generated using ANTLR [5] and JavaCC [47]. The Recoder [62] framework is used for reading and analysing JAVA programs. We use the Dresden OCL parser [31,33] for parsing and type-checking OCL constraints. Finally, the JUnit framework [48] was used for unit testing during development.

\subsection{Structure of the Implementation}

Figure 9 shows the infrastructure of the $\mathrm{KeY}$ tool on the implementation level. The entities shown as cylinders represent external files, while the rectangular ones are programs. The parts rendered with thick lines and bold type are provided by the $\mathrm{KeY}$ project.

The mechanism for instantiating $\mathrm{KeY}$ patterns and idioms as described in Sect. 5, is an extension of the pattern instantiation provided by TogetherCC. Patterns are represented as JAVA programs which construct the required classes, associations, etc., using the TogetherCC API. For KeY patterns, generated entities are annotated 


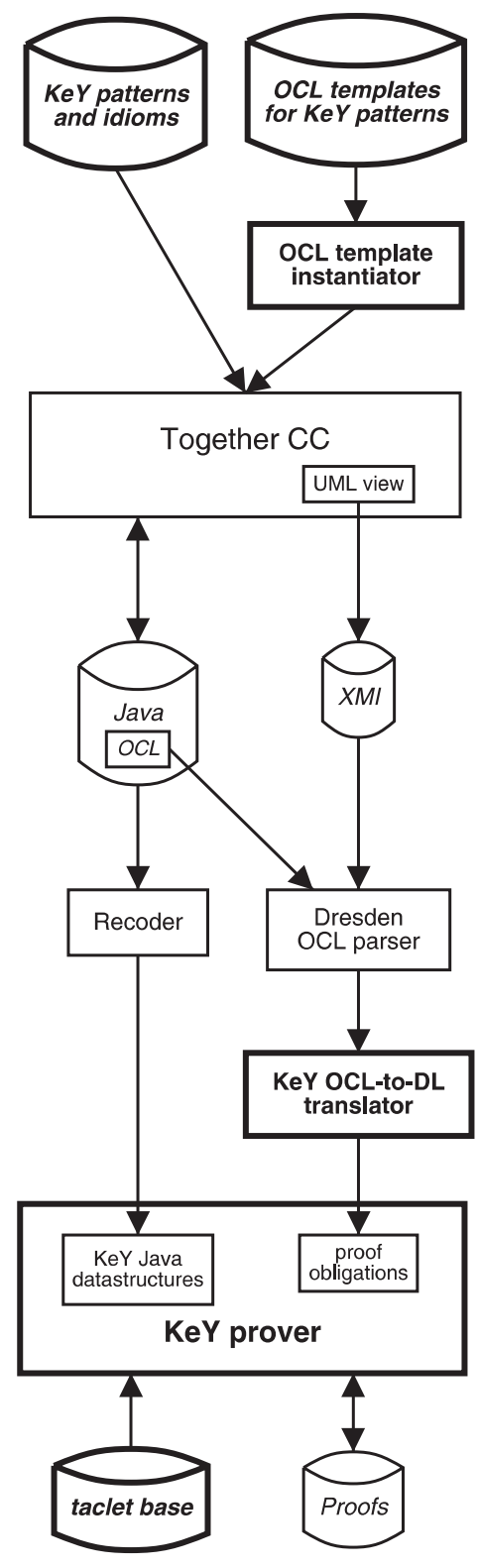

Fig. 9. Infrastructure of the $\mathrm{KeY}$ tool

with OCL constraints. These are generated from OCL template files [12] belonging to each pattern.

Complying with TogetherCC's single source philosophy, OCL constraints are stored as comments in the user's JAVA files.

For syntax and type checking, as well as transformation to dynamic logic, we use the Dresden OCL parser. Type checking requires information about the UML model, which is exported from TogetherCC in XMI format (part of UML standard).

As a consequence of the single source philosophy, the UML model of TogetherCC corresponds one-to-one to the structure of the JAVA implementation.

Alternatively, the model information required by the OCL parser can be extracted without reference to the XMI facility from the JAVA code, using Recoder. Addi- tional information on UML model, for example, associations, is obtained using the TogetherCC API.

When the KeY prover is used to reason about JAVA programs, these are parsed using the Recoder system. Recoder is also used to resolve references, that is, to determine the variable declaration, method declaration, etc., each identifier is referring to.

For the actual proof, different data structures are used, as explained in the following section. Proof obligations are typically generated from OCL constraints. These are translated into dynamic logic formulas (see Sect. 6.2) from the data structures provided by the Dresden OCL parser.

\subsection{Implementation of the theorem prover}

The KeY prover permits automated and interactive construction of proofs for JAVA CARD DL formulas. The central data structure is the proof tree. Its nodes contain sequents, and it is extended by applying rules of the sequent calculus for JAVA CARD DL. In a typical rule application of the sequent calculus, most of the sequent is not changed. To reduce memory consumption, data structures must be used which allow formula representations to be shared among goals and even among branches of the proof tree. Moreover, rule applications usually affect only a small part of the formulas and programs they act upon, so sharing of $s u b$-formulas should also be possible. Sharing implies the use of non-destructive or persistent tree data structures for terms, formulas and programs. Such data structures are ubiquitous in functional and logic programming, but unusual in OO programming. The shared representation of JAVA programs is achieved using a hierarchy of about 250 classes, which are derived from the Recoder data structures.

All proof rules, including those for the automated simplification of goals, are encoded as taclets, see Sect. 8.1 and [16]. Taclets are described by textual representations which are collected in an external file and processed at prover start-up time. There are no "built-in" rules in the KeY prover. Taclets consist of a matching part and an action part. For interactive use, the user specifies a formula or term in the sequent, where a taclet should be applied. This requires finding taclets which match a specific position in the sequent. For automated (heuristic) use, taclets which match anywhere in a sequent are automatically selected. Now matching is a potentially expensive operation, and there is a large number of taclets for the many different JAVA constructs and also for various abstract data types. To speed up the process of finding applicable taclets, these are kept in an indexing structure which permits fast access to a subset of potentially matching taclets. Typically the top-most predicate or function symbol is used to find applicable taclets, in the case of formulas containing programs, the type of the first statement is used. See also [16] for details on the implementation of the taclet mechanism. 


\subsection{Stand-alone versions}

In general, the $\mathrm{KeY}$ tool is designed to be used together with a CASE tool. It was however recognised that parts of the system might be useful independently. It is thus possible to invoke the OCL-to-DL translation of Sect. 6.2 separately, if the UML model is given in XMI format. Furthermore, it is possible to use the KeY prover without a CASE tool. The stand-alone prover parses DL proof obligations from files.

\section{Case studies}

In this section we discuss some case studies that have been used to test the viability of the theoretical approach of $\mathrm{KeY}$ and the implementation of the KeY tool. The case studies can be roughly divided into three categories dependant whether they are concerned with fundamental, security or safety aspects. Table 1 gives an overview of the ongoing and completed case studies.

In the remainder of this section we will briefly summarise each of these studies and point out their main goal.

\subsection{The JAVA collections framework}

The JAvA Collection Framework (JCF) provides an application programming interface (API) plus reference implementation for lists, sets, trees, and related data structures. Many data structures found in JAVA programs are realised via the $\mathrm{JCF}$, hence, it is obvious why they are of main interest for $\mathrm{KeY}$.

Table 1. Completed and ongoing KeY case studies

\begin{tabular}{ccc}
\hline Foundations & Security & Safety \\
\hline $\begin{array}{c}\text { JAVA Collections } \\
\text { Framework }\end{array}$ & PAM & EAST-EEA/Volvo \\
JAVA CARD API & $\begin{array}{c}\text { Secure Information } \\
\text { Flow }\end{array}$ & $\begin{array}{c}\text { Speed Restrictions } \\
\text { for Trains } \\
\end{array}$ \\
& & Command Parser \\
\hline
\end{tabular}

In this case study the informal API documentation of the JCF was formalised in terms of UML/OCL. We investigated then how to refine this quite abstract specification in a way that it can be used to verify the JCF reference implementation with KeY. Such an approach is coherent with the way of developing software by initially creating a model on an abstract level, which is then stepwise refined to the implementational level. On that level, a one-to-one relationship between UML and JAVA classes exists. This refinement process is visualised in Fig. 10.

Usually one wants to assure that (OCL) constraints of refinement level $n+1$ satisfy the constraints of level $n$. But the constraints of level $n$ do not pertain to the possibly changed (usually: enlarged) name space of level $n+1$. Thus, the changes in the model from level $n$ to level $n+1$, together with the constraints of level $n$, are used to compute these constraints in the name space of level $n+1$.

Retrieve relations define the interrelation between the different abstractions. They can be denoted graphically using stereotyped UML dependencies and an additional formal textual description. The specifications obtained from the OCL constraints on the abstract level can be transformed to the concrete level and are used to generate proof obligations (in dynamic logic) that serve to prove the refined model to be a correct refinement.

This technique was successfully applied to parts of the JCF. A complete account of this case study can be found in [64].

\subsection{Specification of the JAVA CARD API}

As part of the KeY project an OCL specification for the JAVA CARD API has been developed [53]. The main purpose of this specification is to support and aid the verification of JAVA CARD programs. The already existing specification written in JML (JAVA Modelling Language) has been used as a starting point for the development of the OCL specification. All parts that were possible to specify with OCL are covered by this specification. Using the KeY system, small parts of the reference implementa-

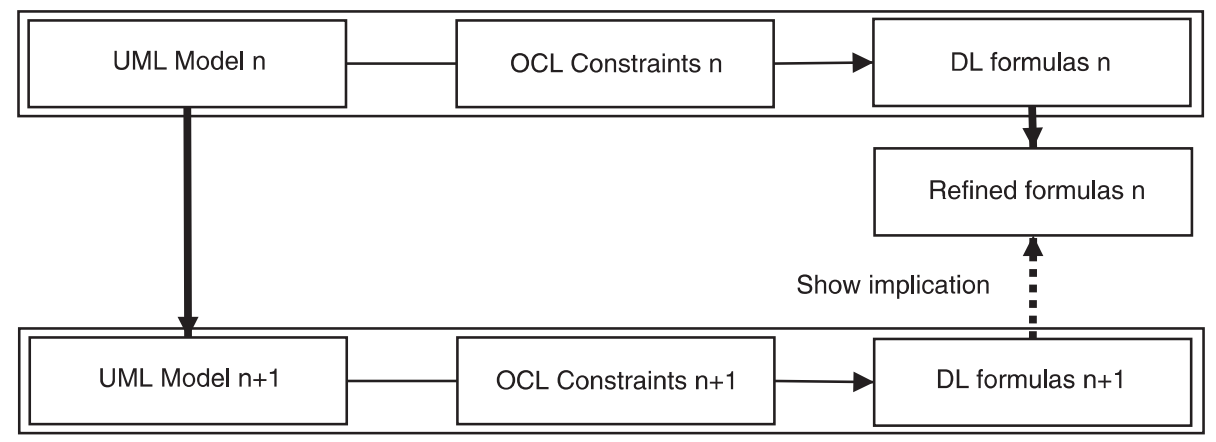

Fig. 10. Schema of a typical refinement step 
tion of the JAVA CARD API has been proved correct w.r.t. this specification.

\subsection{PAM authentication with iButton}

This application allows a Linux user to authenticate himor herself to the system using an iButton ${ }^{4}$ or a smart card instead of a password. The application consists of two parts:

1. The Pluggable Authentication Module (PAM) running on the host system. The module is realised as a PAM library plug-in and, therefore, written in C.

2. The JAVA CARD applet SafeApplet, running on the user's JaVA CARD device.

This time, we consider a genuine JAVA CARD application. The original JAVA CARD applet has however been rewritten and cleaned up for this case study. The redesign of the applet was preceded by an analysis of the system requirements, and guided by questions like:

1. Is the system administrator or the user the owner of the applet PIN code?

2. What are the different deployment states of the applet (how does its life cycle look like)?

3. How can it be ensured that the applet is in a "sound" state when an iButton is ripped out of the reader and how can these "soundness" properties be specified?

The applet life cycle states were captured in a UML state diagrams like the one in Fig. 11. After setting up the state diagrams, the first portions of code (skeleton code) were generated.

During the development process, the OCL specifications of parts of the applet were created, modified, and adjusted with the help of the KeY system. The main challenge are the "rip-out" properties. The problem of specifying those boils down to the general problem of expressing atomicity properties.

4 "iButtons" are particular JAVA CARD devices embedded in a button shaped case, see http://www.ibutton.com/.
It turns out that this requires an extension of JAVA CARD DL with certain modal operators. The suggested modal operator is named $\llbracket \cdot \rrbracket$ (pronounced "throughout"). The formula $\llbracket p \rrbracket \phi$ means that $\phi$ holds after each atomic step in $\operatorname{program} p$.

According to the JAVA CARD specification, an atomic step is an update of a variable or a single object field. A sequence of operations can be bundled to a single atomic step called a transaction by the programmer. Now, it is important to be able to state that $\phi$ does not necessarily hold inside a transaction. The main obstacle here is to capture the semantics and properties of the $\llbracket \cdot \rrbracket$ operator in JAVA CARD DL's calculus.

The "throughout" modality relates to the general problem of specifying and verifying the behaviour of a program in intermediate steps at a low atomicity level and has been well researched in the area of concurrency and reactive systems. E.g. [4] presents a fine-grain semantics for reactive systems and shows how an RSDS specification of such a system can be translated and proved by the SMV model checker. The extension of a dynamic logic calculus for abstract while-programs with the throughout operator was done in the paper [19]. The extension of the JAVA CARD DL calculus to handle transactions is presented in [18]. This extension has also been implemented in the KeY prover and is about to be tested with this and other case studies. For a full paper on the PAM authentication with iButton case study and the development process used, see [56].

\subsection{Analysis of secure information flow}

$\mathrm{KeY}$ was applied to analysis of secure information flow [30]: If there is no information flow from confidential inputs to publicly observable outputs - either directly or indirectly via e.g. control flow - then a program may be considered to be secure. Traditionally this is done by static analyses based on specialized type systems. Although efficient, such approaches need to approximate complex language constructs such as loops, reference types, or exceptions. Verification is not fully automatic, but yields

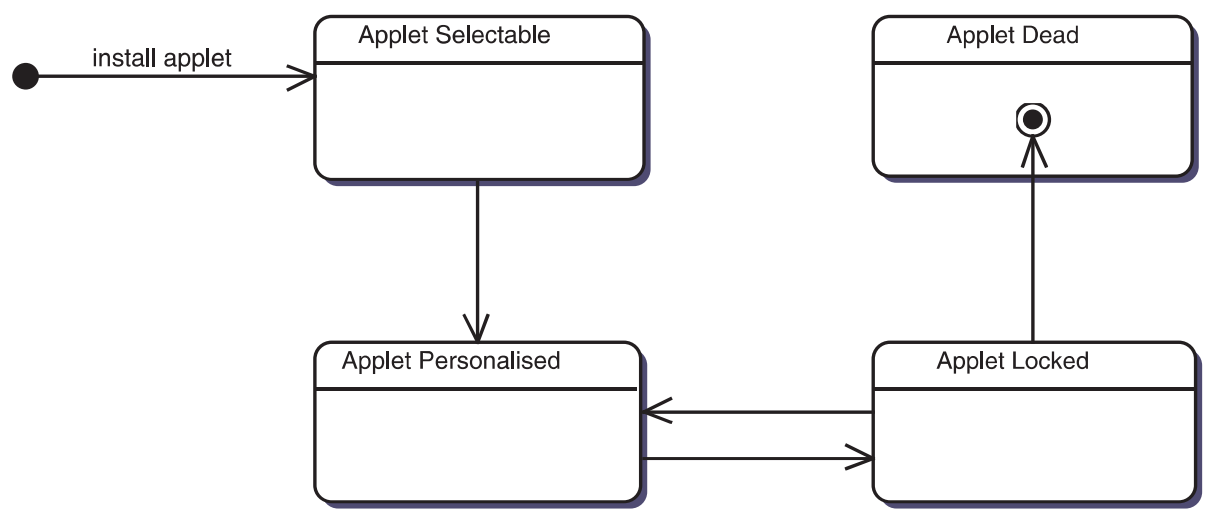

Fig. 11. Life cycle states of the SafeApplet 
higher precision. We were able to prove security and insecurity of programs including advanced features such as method calls, loops, and object types for the target language JAVA CARD. In addition, we can express declassification of information. Secure information flow can be characterized by relatively simple proof obligations so that the degree of automation is higher than for full property verification.

\subsection{Formal language for design requirements in automotive domain}

EAST-EEA (Embedded Electronic Architecture) is an ITEA project to enable electronic integration of automotive platforms through definition of an open architecture. With Volvo Technology AB we work on a UML 2.0-based formal language for design requirements.

\subsection{Computation of speed restrictions of trains}

Several hundred trains run at any given time on the network of the German railway company Deutsche Bahn AG $(D B)$. Strict compliance by the train to numerous restrictions such as speed limits, signals and brake distances, is an absolute safety requirement.

The train drivers are handed out a schedule in printed or electronic form containing the speed restrictions and other additional information along the different way (track) points of a route. These schedules are computed for each train-route combination and consist of headers and tables. The headers contain general information about the route and required technical features of the trains, for example the required minimal brake power. Track point dependant information like speed restrictions or signals are listed in the tables. The 'schedule' approach allows a flexible respond to the available technology of concrete trains (e.g. tilting), in contrast to the rather rigid road traffic speed restrictions.

Responsible for the schedule computation is the software system Satzerstellung betrieblicher Fahrplanunterlagen $(S b F)$ developed by DBSystems, which served as starting point for the present study.

DBSystems provided us with a current version of $S b F$ and the product description in natural language, which described among others the speed computation algorithm. The program SbF itself is written in Smalltalk consisting of estimated seven hundred classes from which around eighty are concerned with the speed and brake power computation. The latter classes had to be crosstranslated into JAVA, which is required by KeY. The aim is to achieve a verified JAVA program, whose behaviour can be compared on runtime with the original program. Consequently not the correctness of the original program will be proven, but the correctness of the computed booktables via the verified reference implementation.

The first step, was to formalise the product specification in UML/OCL. The resulting analysis model abstracts away from all details of the concrete implementation, for example, modeling the infrastructure as shown in Fig. 12 required eleven classes, whereas the actual implementation makes use of more than twenty five classes. Incrementing the level of detail towards the actual implementation involves several well organised refinement steps, which are currently worked out using the refinement technique as described in [64], see Sect. 10.1.

As an intermediate result of writing the formal specification we discovered some ambiguities and incompleteness of the product specification and surprisingly also

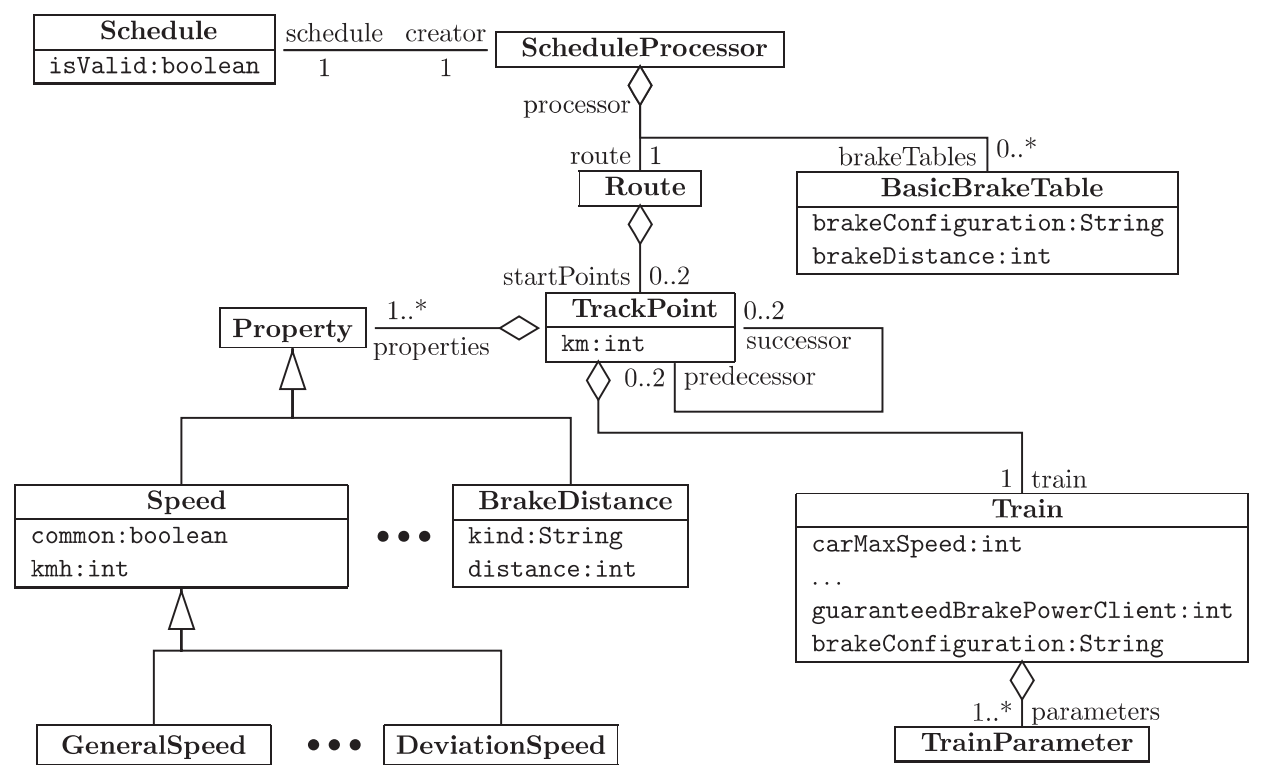

Fig. 12. Infrastructure model on the analysis level 
runtime inefficiencies. The verification of $S b F$ is under way.

\subsection{A command parser for chemical analysis devices}

The specification and verification of a command parser used in a series of chemical analysis devices is done in cooperation with Agilent Technologies and the Institut für Technik der Informationsverarbeitung of the University of Karlsruhe.

Agilent produces several chemical analysis devices used among others for research or medical purposes. The different product lines share a common subset of modules. One of these modules is a command parser, which is for example used to parse steering commands of injection pumps.

The aim is to specify the parser in UML/OCL and verify its reimplementation in JAVA. The verified reimplementation is then automatical translated to $\mathrm{C}++$ code using the tool GeneralStore.

\subsection{Summary}

The case studies discussed here differ considerably with respect to the nature of the target programs, but also with respect to their objectives. They show that the KeY approach is flexible enough to cope with varying demands. The case studies, respectively, the intermediate results obtained so far, demonstrate the viability and usability of the KeY approach.

\section{Current state and future work}

\subsection{Current state}

The core of the $\mathrm{KeY}$ tool is finished. All features of JAVA CARD including the transaction mechanism are supported by the tool and it is, with a few minor restrictions, already successfully applicable. ${ }^{5}$ The case studies described in Sect. 10 prove this. They also show that it is possible to verify the average JAVA method (consisting of about 10 to 20 lines of code) within a few minutes. Often, the KeY prover can even automatically establish correctness if the code does not contain loops (in this case the user has to provide a loop invariant interactively).

To improve the usability of the KeY tool we have integrated an authoring tool for OCL constraints [41]. This tool offers assistance in generating specifications and helps to understand OCL constraints by rendering them automatically in natural language. We believe that the integration of such a tool helps to overcome reservations against formal methods.

\footnotetext{
5 The KeY tool can be downloaded at http://download.keyproject.org/. The version available for download supports all JAVA CARD features except for class initialisation which is currently implemented.
}

Another important aspect is the support of proof reuse in the $\mathrm{KeY}$ tool [50]. This technique diminishes the amount of work spent with verification after a (minor) change of the specification or implementation. We count this as an essential point since one of the prejudices against program verification is that it is too costly to be ever usable in practice.

Proof re-use makes it feasible to check automatically and periodically whether the implementation still complies with the properties expressed in the OCL constraints. The situation is similar to automatic and periodic runs of unit tests, a proven best practice in software development. Periodical checks prevent specifications from becoming outdated, which is a major (and common) problem when specifications are merely available as informal text.

\subsection{Future work}

In practice, proof attempts often fail simply because the implementation does not satisfy the specification. In such a situation a tool assisting the user in identifying and solving errors in the implementation would be very valuable. A theoretical framework for counter-example generation based on abstract data types is presented in [1]. The usefulness of this approach has already been shown in a prototype which is based on a model generation theorem prover [36]. We would like to adapt this tool to our setting and integrate it into the $\mathrm{KeY}$ tool.

One obvious direction for future work includes support of UML diagram types other than class diagrams, such as state chart or sequence diagrams. This would allow to specify temporal behaviour of programs, which is not possible in class diagrams.

To make software verification scale up to larger programs, it is necessary to have a module concept that makes it possible to independently verify the modules of a program. Modules are well understood for imperative languages and are supported by several languages. Unfortunately, object-oriented languages including JAVA lag somewhat behind. One of our next research efforts will be to look for a module concept for JAVA that is compatible with the requirements of formal verification.

We are aware that formal verification is only one option among many formal methods (and perhaps a rather extreme one). Other approaches, such as abstract interpretation, first order model checking, static analysis, extended static checking, etc., should be integrated into the $\mathrm{KeY}$ tool.

Finally, we would like to improve the user interface to the theorem prover in a fundamental way: recall that one can view verification in $\mathrm{KeY}$ as symbolic program execution. Hence, one may see a single branch in the proof tree as one program execution with symbolic start values. We intend to reformulate verification as much as possible within the well-established paradigm of symbolic source code debugging. We think that a remod- 
elling of formal verification as "abstract debugging" will not only increase acceptance of verification, but will result in a massive improvement of efficiency: such elements of modern debuggers as break points, watches, spy points, inspectors, and navigation aides make eminent sense within the symbolic execution paradigm, too. In addition, one can create views of formal proofs that allow the engineer to interact with the prover more easily. For example, the current state of symbolic program execution (object instances, values of attributes and local variables, object references, method call stack, active command, etc.) can be extracted from the JAVA CARD DL formulas in an open proof goal and presented like in a conventional source code debugger. Such a debugger based on theorem proving goes beyond current debugging tools, because full first-order logic is available as an assertion language.

\section{Conclusion}

What sets the $\mathrm{KeY}$ tool apart from other efforts in formal software specification and verification is the systematic attempt to conceive a formal technique as an extension of established, industrial methods of software development.

Unfortunately, parts of the formal methods community in the past have denounced popular industrial software development methods as unscientific and, hence, unworthy of consideration. Such views are based on a lack of knowledge about industrial software production and the conflation of "scientific" with "formal". In contrast to this, we believe that formal methods must be tightly integrated with conventional development processes in order to be immediately useful to developers and designers. We see this as a prerequisite to be fulfilled before formal methods can possibly catch on.

The most visible point of the philosophy just sketched is the interface of the $\mathrm{KeY}$ tool, which appears to be a state-of-the-art, although conventional CASE tool. Formal specifications can be added anytime during design and development without having to change the tool or paradigm. Machine assistance in generating formal specification in the form of $\mathrm{KeY}$ idioms and patterns help users to get started. The resulting high degree of integration with a commercial CASE tool is where KeY goes beyond other recent approaches that aim at integration of software engineering with formal methods $[29,52,66] .{ }^{6}$

Integration of informal and formal methods is one of the corner stones of the $\mathrm{KeY}$ approach. A second one is the consequent choice and design of the formal tools so as to maximise their usability. For example, the program logic JAVA CARD DL is transparent with respect to the tar-

\footnotetext{
6 This does, of course, not mean that these projects are redundant: they cover different languages, domains, and technologies than $\mathrm{KeY}$.
}

get language and supports symbolic execution as a proof paradigm. We are convinced that the pragmatics and usability of formal tools are as important as their soundness and theoretical adequacy.

We would like to stress that the integration and scaling-up of our formal method spawned a considerable number of theoretically interesting questions (resulting in technical papers such as $[15,17-21])$. Likewise, formalisation of UML and OCL led to clarifications and extensions $[6-9,11]$.

Finally, we turn to a brief discussion of the most frequently heard counter argument against software verification, which can be paraphrased like this: "full functional verification will never be a push button technology; but this is a sine qua non for formal methods to catch on in industry. Unless you produce something like a model checker as used for verification of hardware and system designs, you will never prevail."

We agree that formal software verification is extremely unlikely to become fully automated, however, this is a red herring, because the assessment above is based on a (at least) twofold misunderstanding: first, the stumbling block for industrial users when applying formal methods is not interactivity. The problem is that current formal approaches are idiosyncratic and require special skills. The usability threshold is simply very high. Widely established methods and tools such as symbolic debuggers, most testing methods, code reviews, etc., are all far from being automated. They are accepted, because they are useful, integral parts of processes, and they can be mastered with reasonable effort. Second, so-called "push button" technologies, notably symbolic model checking, are far from being automated either: system requirements have to be captured formally in a temporal logic, suitable abstractions must be discovered, model checkers have to be tweaked to cope with large problems, etc.

In summary, the real challenge for formal methods in software development is to make them useful for as many people as possible. This is what the KeY project is about.

Acknowledgements. We would like to thank numerous students for their contributions to the implementation of the KeY tool. We are indebted to our former colleague Elmar Habermalz for his contributions in the early stages of the KeY project. We also thank the reviewers for pointing out a number of inaccuracies and additional references.

\section{References}

1. Ahrendt W (2002) Deductive search for errors in free data type specifications using model generation. In: Voronkov A (ed) Automated Deduction - CADE-18, 18th International Conference on Automated Deduction, Copenhagen, Denmark, LNCS, vol 2392. Springer-Verlag

2. Ahrendt W, Baar T, Beckert B, Giese M, Habermalz E, Hähnle R, Menzel W, Schmitt PH (2000) The KeY approach: Integrating object oriented design and formal verification. In: Ojeda-Aciego M, de Guzmán IP, Brewka G, Pereira LM (eds) Proc. 8th European Workshop on Logics in AI (JELIA), LNCS, vol 1919. Springer-Verlag, pp 21-36, Oct. 
3. Ahrendt W, Baar T, Beckert B, Giese M, Hähnle R, Menzel W, Mostowski W, Schmitt PH (2002) The KeY system: Integrating object-oriented design and formal methods. In: Kutsche R-D, Weber H (eds) Fundamental Approaches to Software Engineering (FASE), Part of Joint European Conferences on Theory and Practice of Software, ETAPS, Grenoble, LNCS, vol 2306. Springer-Verlag, pp 327-330

4. Androutsopoulos K (2002) Using SMV to model check RSDS specifications. Technical Report TR-02-07, King's College of London, Department of Computing Science

5. ANTLR homepage. At http://www.antlr.org/

6. Baar T (2002) How to ground meta-circular OCL descriptions: A set-theoretic approach. In: Clark T, Evans A, Lano K (eds) Proceedings, Fourth Workshop on Rigorous Object-Oriented Methods, London

7. Baar T (2003) The definition of transitive closure with ocl: Limitations and applications. In: Proceedings, Fifth Andrei Ershov International Conference, Perspectives of System Informatics, Novosibirsk, Russia, LNCS, vol 2890. Springer, pp 358-365, July

8. Baar T (2003) Über die Semantikbeschreibung OCL-artiger Sprachen. $\mathrm{PhD}$ thesis, Fakultät für Informatik, Universität Karlsruhe. ISBN 3-8325-0433-8, Logos Verlag, Berlin

9. Baar T (2004) Metamodels without metacircularities. L'Objet. To appear

10. Baar T, Beckert B, Schmitt PH (2001) An extension of Dynamic Logic for modelling OCL's @pre operator. In: Proceedings, Fourth Andrei Ershov International Conference, Perspectives of System Informatics, Novosibirsk, Russia, LNCS, vol 2244. Springer, pp 47-54

11. Baar T, Hähnle R (2000) An integrated metamodel for OCL types. In: France R, Rumpe B, Whittle J (eds) Proc. OOPSLA 2000 Workshop Refactoring the UML: In Search of the Core, Minneapolis/MI, USA, Oct.

12. Baar T, Hähnle R, Sattler T, Schmitt PH (2000) Entwurfsmustergesteuerte Erzeugung von OCL-Constraints. In: Mehlhorn K, Snelting G (eds) Softwaretechnik-Trends, Informatik Aktuell, pp 389-404. Springer-Verlag, Sept. In German.

13. Balser M, Reif W, Schellhorn G, Stenzel K, Thums A (2000) Formal system development with KIV. In: Maibaum T (ed) Fundamental Approaches to Software Engineering, LNCS, vol 1783. Springer-Verlag

14. Beck K (1999) Embracing change with Extreme Programming. Computer 32:70-77, Oct.

15. Beckert B (2001) A dynamic logic for the formal verification of Java Card programs. In: Attali I, Jensen T (eds) Java on Smart Cards: Programming and Security. Revised Papers, Java Card 2000, International Workshop, Cannes, France, LNCS, vol 2041. Springer-Verlag, pp 6-24

16. Beckert B, Giese M, Habermalz E, Hähnle R, Roth A, Rümmer P, Schlager S (2004) Taclets: A new paradigm for writing theorem provers. Revista De La Real Academia De Ciencias Exactas, Fisicas Y Naturales. To appear.

17. Beckert B, Keller U, Schmitt PH (2002) Translating the Object Constraint Language into first-order predicate logic. In: Proceedings, VERIFY, Workshop at Federated Logic Conferences (FLoC), Copenhagen, Denmark. Available at http://i12www.ira.uka.de/ key/doc/2002/BeckertKeller Schmitt02.ps.gz

18. Beckert B, Mostowski W (2003) A program logic for handling JAVA CARD's transaction mechanism. In: Pezzè M (ed) Proceedings, Fundamental Approaches to Software Engineering (FASE) Conference, LNCS, vol 2621. Warsaw, Poland. Springer, pp 246-260, April

19. Beckert B, Schlager S (2001) A sequent calculus for firstorder dynamic logic with trace modalities. In: Gorè R, Leitsch A, Nipkow T (eds) Proceedings, International Joint Conference on Automated Reasoning, Siena, Italy, LNCS vol 2083. Springer, pp 626-641

20. Beckert B, Schlager S (2004) Software verification with integrated data type refinement for integer arithmetic. In: Proceedings, International Conference on Integrated Formal Methods, Canterbury, UK, LNCS. Springer. To appear

21. Beckert B, Schmitt PH (2003) Program verification using change information. In: Proceedings, Software Engineering and Formal Methods (SEFM), Brisbane, Australia. IEEE Press, pp 91-99

22. Boehm BW (1988) A spiral model of software development and enhancement. IEEE Computer 21(5):61-72

23. Borland Together homepage.

At http://www.borland.com/together/index.html

24. Breu R, Grosu R, Huber F, Rumpe B, Schwerin W (1997) Towards a precise semantics for object-oriented modeling techniques. In: Bosch J, Mitchell S (eds) Object-Oriented Technology, ECOOP'97 Post Conference Workshop Reader, Jyväskylä, Finland, LNCS, vol 1357. Springer-Verlag

25. Brucker AD, Wolff B (2002) HOL-OCL: Experiences, consequences and design choices. In: Jézéquel J-M, Hussmann H, Cook S (eds) UML 2002: Model Engineering, Concepts and Tools, LNCS, vol 2460. Springer-Verlag, pp 196-211

26. Bubel R, Hähnle R (2003) Formal specification of securitycritical railway software with the KeY system. In: Arts T, Fokkink W (eds) Proceedings, Eighth International Workshop on Formal Methods for Industrial Critical Systems (FMICS), Electronic Notes in Theoretical Computer Science, vol 80. Elsevier

27. Chen Z (2000) Java Card Technology for Smart Cards: Architecture and Programmer's Guide. Java Series. AddisonWesley, June

28. Cook S, Daniels J (1994) Designing Object Systems: ObjectOriented Modelling with Syntropy. The Object-Oriented Series. Prentice Hall

29. Crocker D (2002) Perfect Developer: A tool for rigorous object-oriented software development. In: Clark T, Evans A, Lano K (eds) Proc. Fourth Workshop on Rigorous ObjectOriented Methods, London

30. Darvas A, Hähnle R, Sands D (2003) A theorem proving approach to analysis of secure information flow. In: Gorrieri $\mathrm{R}$ (ed) Workshop on Issues in the Theory of Security (WITS). IFIP WG 1.7, ACM SIGPLAN and GI FoMSESS

31. Dresden-OCL homepage. At http://dresden-ocl. sourceforge.net/

32. Evans A, Bruel J-M, France R, Lano K, Rumpe B (1998) Making UML precise. In: Andrade L, Moreira A, Deshpande A, Kent S (eds) Proceedings of the OOPSLA'98 Workshop on Formalizing UML. Why? How?

33. Finger F (2000) Design and implementation of a modular OCL compiler. Diplomarbeit, Technische Universität Dresden, Fakultät für Informatik, Mar.

34. Fowler M, Scott K (1997) UML Destilled. Applying the Standard Object Modeling Language. Addison-Wesley

35. France R (1999) A problem-oriented analysis of basic UML static requirements modeling concepts. In: Proceedings of the 1999 ACM SIGPLAN conference on Object-oriented programming, systems, languages, and applications. ACM Press, pp $57-69$

36. Fujita H, Hasegawa R (1991) A model generation theorem prover in KL1 using a ramified-stack algorithm. In: Furukawa K (ed) Proceedings 8th International Conference on Logic Programming, Paris/France. MIT Press, pp 535-548

37. Gamma E, Helm R, Johnson R, Vlissides J (1995) Design Patterns: Elements of Reusable Object-Oriented Software. Addison-Wesley, Reading/MA

38. Giese M (2001) Incremental closure of free variable tableaux. In: Goré, R., Leitsch A, Nipkow T (eds) Proc. Intl. Joint Conference on Automated Reasoning (IJCAR), Siena, Italy, LNCS, vol 2083. Springer-Verlag, pp 545-560

39. Giese M (2003) Taclets and the KeY prover. In: Lüth C, Aspinall D (eds) Intl., Workshop on User Interfaces for Theorem Provers, UITP 2003, Rome, Italy. Arcane, Rome, pp 74-80. Also as Tech. Report 189, Inst. f. Informatik, Albert-LudwigsUniversität, Freiburg

40. Habermalz E (2000) Interactive theorem proving with schematic theory specific rules. Technical Report 19/00, Fakultät für Informatik, Universität Karlsruhe. Available at http://i12www . ira.uka.de/ key/doc/2000/stsr.ps.gz

41. Hähnle R, Johannisson K, Ranta A (2002) An authoring tool for informal and formal requirements specifications. In: Kutsche R-D, Weber H (eds) Fundamental Approaches to Software Engineering (FASE), Part of Joint European Confer- 
ences on Theory and Practice of Software, ETAPS, Grenoble, LNCS, vol 2306. Springer-Verlag, pp 233-248

42. Harel D (1984) Dynamic logic. In: Gabbay D, Guenthner F (eds) Handbook of Philosophical Logic, volume II: Extensions of Classical Logic, chapter 10. Reidel, Dordrecht, pp 497-604

43. Harel D, Kozen D, Tiuryn J (2000) Dynamic Logic. MIT Press

44. Holzmann GJ (2001) Economics of software verification. In: Proc., Workshop on Program Analysis for Software Tools and Engineering, Snowbird, Utah, USA, ACM, June

45. Hutter D, Langenstein B, Sengler C, Siekmann JH, Stephan W (1996) Deduction in the Verification Support Environment (VSE). In: Gaudel M-C, Woodcock J (eds) Proceedings, Formal Methods Europe: Industrial Benefits Advances in Formal Methods. Springer

46. Jacobson I, Rumbaugh J, Booch G (1999) The Unified Software Development Process. Object Technology Series. Addison-Wesley, Reading/MA

47. JavaCC homepage. At http://www.webgain.com/products/java_cc/

48. JUnit homepage.

At http://junit.sourceforge.net/

49. Keller U (2002) Übersetzung von OCL-Constraints in Formeln einer Dynamischen Logik für Java. Diplomarbeit, Fakultät für Informatik, Universität Karlsruhe. In German

50. Klebanov V (2003) Proof Re-Use in Java Software Verification. Diplomarbeit, Fakultät für Informatik, Universität Karlsruhe

51. Kozen D, Tiuryn J (1990) Logics of programs. In: van Leeuwen J (ed) Handbook of Theoretical Computer Science, volume B: Formal Models and Semantics, chapter 14. The MIT Press, pp 789-840

52. Lano K, Clark D, Androutsopoulos K (2002) Formalising inter-model consistency of the UML. In: Kuzniarz L, Reggio G, Sourrouille JL, Huzar Z (eds) Blekinge Institute of Technology, Research Report 2002:06. UML 2002, Model Engineering, Concepts and Tools. Workshop on Consistency Problems in UML-based Software Development. Workshop Materials. Department of Software Engineering and Computer Science, Blekinge Institute of Technology, pp 133-148

53. Larsson D, Mostowski W (2004) Specifying Java Card API in OCL. In: OCL 2.0 Workshop at UML 2003, ENTCS. Elsevier. To appear

54. Mellor SJ, D'Souza D, Clark T, Evans A, Kent S (2001) Infrastructure and Superstructure of the Unified Modeling Language 2.0 (Response to UML2.0 RfP). Technical report, Submission to the OMG

55. Meyer B (1997) Object-Oriented Software Construction. Prentice-Hall, Englewood Cliffs, second edition

56. Mostowski W (2002) Rigorous development of JavaCard applications. In: Clark T, Evans A, Lano K (eds) Proc. Fourth Workshop on Rigorous Object-Oriented Methods,
London. Available at http://www.cs.chalmers.se/ $\sim$ woj/ papers/room2002.ps.gz

57. Response to the UML OCL RfP (2002) June. OMG document ad/2002-05-09

58. Object Modeling Group (2003) Unified Modelling Language Specification, version 1.5, Mar.

59. Owre S, Rajan S, Rushby J, Shankar N, Srivas M (1996) PVS: Combining specification, proof checking, and model checking. In: Alur R, Henzinger TA (eds) Computer-Aided Verification, CAV '96, LNCS, vol 1102. Springer-Verlag, pp 411-414, July/August

60. Paulson LC (1994) Isabelle: a generic theorem prover, LNCS, vol 828. Springer-Verlag

61. Pratt VR (1977) Semantical considerations on Floyd-Hoare logic. In: Proceedings, 18th Annual IEEE Symposium on Foundation of Computer Science

62. Recoder homepage. http://recoder. sourceforge.net/

63. Richters M (2002) A Precise Approach to Validating UML Models and OCL Constraints, BISS Monographs, vol 14. Logos Verlag. PhD thesis, Universität Bremen

64. Roth A (2002) Deduktiver Softwareentwurf am Beispiel des Java Collections Frameworks. Diplomarbeit, Fakultät für Informatik, Universität Karlsruhe, June. In German

65. Schmitt PH (2001) A model theoretic semantics of OCL. In: Beckert B, France R, Hähnle R, Jacobs B (eds) Proceedings, IJCAR Workshop on Precise Modelling and Deduction for Object-oriented Software Development, Siena, Italy. Technical Report DII 07/01, Dipartimento di Ingegneria dell'Informazione, Università degli Studi di Siena, pp 43-57

66. Snook C, Wheeler P, Butler M (2003) Preliminary tool extensions for integration of UML and B. IST-2000-30103 project deliverable D4.1.2. Available at http://www.keesda.com/pussee/

67. Stenzel K (2001) Verification of java card programs. Technical report 2001-5, Institut für Informatik, Universität Augsburg, Germany. Available at http://www. Informatik. Uni-Augsburg.de/swt/fmg/papers/

68. Sun Microsystems, Inc. (2001) Java Card 2.0 Language Subset and Virtual Machine Specification, Palo Alto/CA, Oct.

69. Sun Microsystems, Inc. (2002) Java Card 2.2 Platform Specification, Palo Alto/CA, USA, Sept.

70. von Oheimb D (2000) Axiomatic semantics for Java ${ }^{\text {light. In: }}$ Drossopoulou S, Eisenbach S, Jacobs B, Leavens GT, Müller P, Poetzsch-Heffter A (eds) Proceedings, Formal Techniques for Java Programs, Workshop at ECOOP'00, Cannes, France

71. von Oheimb D (2001) Analyzing Java in Isabelle/HOL. $\mathrm{PhD}$ thesis, Institut für Informatik, Technische Universität München, Jan.

72. Warmer J, Kleppe A (1999) OCL: The constraint language of the UML. Journal of Object-Oriented Programming, 12(1):10-13,28, Mar. 